\title{
MODELACIÓN MATEMÁTICA EN ESTUDIO DE AGRO- CADENAS: UNA REVISIÓN DE LITERATURA
}

\author{
Dairo Steven Muñoz-Pinzón1, Andrés Polo-Roa1, Elvira Julieth Sierra-Mantilla1', Diego Fernando \\ Rueda-Uribe $^{1}$
}

${ }^{1}$ Fundación Universitaria Agraria de Colombia

Correo de correspondencia: dairo.munoz@outlook.com

\begin{abstract}
RESUMEN
El sector agrícola es el eje fundamental que mueve la economía del mundo, permite la generación de productos agrícolas y pecuarios para el abastecimiento de pequeñas y grandes ciudades. En el presente trabajo se realizó una revisión de literatura sobre la aplicación de la modelación matemática en el estudio de las Agrocadenas durante los últimos 20 años. Se concluye del estudio que, existe un interés bastante grande por la comunidad académico-científica por fortalecer el sector agrícola en diferentes países como Estados Unidos, Brasil, india y Holanda entre otros.La modelación matemática se ha convertido en una herramienta de gran utilidad para la solución de problemas latentes en la agro-cadenas, facilita el tratamiento de datos y la toma de decisiones complejas, principalmente durante el diseño de cadena, el abastecimiento de producto y control de costos, tiempos de entrega e impactos ambientales, entre otras variables importantes.
\end{abstract}

Palabras clave: Cadena de suministro; Modelos matemáticos; Revisión de literatura; Agrocadenas.

Recibido: 02 de Diciembre de 2019. Aceptado: 28 de Mayo de 2020.

Received: December 02, 2019. Accepted: May 28, 2020.

\section{ANALYSIS OF THE USE OF MATHEMATICAL MODELS FOR THE STUDY OF SUPPLY CHAINS: A REVIEW OF THE LITERATURE}

\begin{abstract}
The agricultural sector is the fundamental axis that moves the world economy, it allows the generation of agricultural and livestock products to supply small and large cities. An approach used to strengthen the sector is the study of agricultural supply chains (agro-chains) based on mathematical modeling, that allows data processing and facilitates strategic, tactical or operational decision-making. We conducted a review of the literature on the application of mathematical models in the study of agricultural chains during the last 20 years. The study concludes that there is a fairly great interest by the academic-scientific community to strengthen the agricultural sector in different countries such as the United States, Brazil, India and the Netherlands, among others. Mathematical modeling has become a very useful tool for solving latent problems in agro-chains, it facilitates data processing and complex decision-making, mainly during chain design, product supply and control of costs, delivery times and environmental impacts, among other important variables.
\end{abstract}

Keywords: Supply chain; Mathematical models; Literature review; Agricultural chains.

Cómo citar este artículo: D. Muñoz, A. Polo, E. Sierra, D. Rueda. "Modelación matemática en estudio de agrocadenas: una revisión de literatura", Revista Politécnica, vol.16, no.31 pp.110-137, 2020. DOI: 10.33571/rpolitec.v16n31a9 


\section{INTRODUCCIÓN}

En el sector agrícola existen problemas complejos que requieren el uso de herramientas y tecnologías para su solución y así hacer que los sistemas agrícolas funcionen adecuadamente dando cumplimiento a las necesidades de cada país. Durante los últimos años, existe una evidente tendencia en diferentes países por fortalecer el sector agrícola, esto se debe a que, es una de las divisiones más importantes para el desarrollo sostenible. En países en vía de desarrollo que emplean la agricultura como su eje productivo, el incremento de la productividad en sus cadenas de abastecimiento es un factor elemental para generar progreso. Este incremento se logra con innovación tecnológica y el acceso a nuevos mercados [1]. Es necesario generar estrategias innovadoras y sostenibles para que los sistemas de abastecimiento agrícolas operen correctamente [2].

La optimización de los recursos en la producción agrícola es indispensable [3] para reducir costos y aumentar a su vez las utilidades operacionales; sin embargo, las agro-cadenas son complejas por el tipo de productos que se manejan, muchos productos como frutas y verduras son perecederos [4] y se ven afectados por las variaciones de temperatura, maduración excesiva y deterioro mecánico de transporte [5], es necesario conservar su inocuidad [6]. Según OCDE/FAO [7] el $28 \%$ de la superficie agrícola cultiva productos que se desperdician, se despilfarran mundialmente $1,600,000,0000$ de toneladas por año de producto, lo que genera cerca de 3300 millones de toneladas de $\mathrm{C} 02$ de gases efecto invernadero. Por otra parte, cuándo se trata de animales o subproductos de origen animal se debe evaluar el bienestar animal como la ubicación de sitios de descanso para animales transportados hacia plantas de beneficio [8]. Adicionalmente, en el estudio de las agro-cadenas debe tenerse en cuenta políticas gubernamentales que promueven la participación de grandes, medianos y pequeños eslabones que la componen [9]. El estudio de las agro-cadenas (cadenas agrícolas) no obedece solamente a disminuir costos o incrementar utilidades, es necesario evaluar la logística necesaria para disminuir desperdicios, impactos ambientales y/o sociales durante el proceso [10], [11], [12] y [13].

Algunos problemas asociados al estudio de las cadenas de suministro agrícolas son considerados de gran complejidad, e incluso pueden contener un alto grado de incertidumbre; Inclusive, algunos autores han optado por la medición de indicadores financieros como el VPN o valor actual neto [14] y consideraciones sobre planificación fiscal como los impuestos [15] para la toma de decisiones con mayor precisión. La modelación y las herramientas tecnológicas permiten procesar y analizar los datos con precisión, para así encontrar soluciones "exactas" a problemas complejos [16]. Durante muchos años, diferentes autores se han dedicado modelar matemáticamente el funcionamiento de los sistemas de producción y logísticos agrícolas [17] para el análisis y la toma de decisiones oportuna dada su exactitud en el procesamiento de la información.

Considerando la diversidad de problemas asociados al estudio de agro-cadenas y la importancia de la modelación matemática para representar los sistemas logísticos y facilitar la toma de decisiones, el presente estudio tiene como objetivo realizar una revisión de literatura sobre la aplicación de la modelación matemática en el estudio de las Agrocadenas durante los últimos 20 años. Se resalta que, en literatura no se evidencia una revisión que muestre como se ha aplicado la modelación matemática para estudio de las agrocadenas. Es importante, saber cómo se ha abordado los problemas latentes en la agrocadenas con la modelación matemática, para tomar como punto de partida en futuras investigaciones que se puedan desarrollar en este sector.

El documento está organizado como se describe a continuación: En la segunda sección se presenta la metodología de trabajo para realizar la revisión de literatura; la tercera sección muestra un análisis bibliométrico sobre los trabajos consultados; en la cuarta sección se pueden ver los resultados de la revisión de literatura; y finalmente, la quita sección muestra las conclusiones del estudio.

\section{METODOLOGÍA}

Se realizó una sistematización de la información para la búsqueda y extracción de teoría encontradas en la literatura. Durante el proceso, se utilizaron bases de datos académicas como Proquest, Science-Direct, Scielo y Scopus, para la consulta de artículos producto de investigación asociados estudio agrocadenas a partir de la modelación 
matemática. Fue necesario definir a partir de la experticia de los autores palabras clave como: Food supply chain, Optimization of agricultural, Agricultural supply chain, Linear programming agricultural, Vegetables and fruits distributions planning, Optimization of fresh food supply chain, Distribution of vegetables and fruits, Modelling food logistic, Farm management optimization, Mathematical model optimizing agriculture supply chain, Optimizing fresh food logistic, Fruits transportation planning. Se obtuvo un resultado inicial de búsqueda 605,027 documentos, por lo que fue necesario depurar la información.

Se decidió consultar solo los artículos que fueran publicados desde el año 2000, esto considerando que para los autores es de interés ver el comportamiento de la investigación desde inicios del siglo XXI. Como resultado de este primer filtro, se obtuvo un total de 496,772 documentos, por lo que fue necesario seguir estableciendo criterios de filtración. Se procedió con la lectura y descarga de los artículos; sin embargo, solamente se tuvieron en cuenta los primeros 300 artículos que se visualizaban en las bases de datos, esto por palabra clave, esto debido a que a partir de la visualización del articulo 300 los temas que abordaban no eran tan relevantes para el presente estudio.

Este ejercicio permitió la selección de los artículos más relevantes con el objeto de estudio. Aplicando estos criterios se descargó de las bases de datos una totalidad de 268 artículos, los cuales, fueron leídos para extraer teorías asociadas al tema a partir de la sistematización de información. Durante la lectura de los documentos se descartaron 68 documentos debido a que en sus estudios no incluían el uso de un modelo matemático o simplemente no estaban relacionados como sector agrícola. Esto condujo a incluir en el estudio 200 documentos que cumplen con la temática planteada.

Para la extracción de teorías se construyó una matriz de sistematización, la cual se dividió por subtemáticas para poder organizar la información, entre estas temáticas se encuentra el subsector, las variables de estudio, el tipo de modelos que se utilizan, el nivel de aplicación, entre otras. Esta división se hizo con el propósito de presentar de forma ordenada los resultados del estudio. Los resultados y el análisis de las teorías asociadas al objeto de estudio se presentan en los apartados siguientes del documento.

\section{ANÁLISIS BIBLIOMÉTRICO}

Se realizó un análisis biblio-métrico con las palabras clave de los doscientos (200) artículos incluidos consultados. Esto se hizo con ayuda del Software Vosviewer, el cual, permitió ver la relación entre las temáticas que se abordan cuando se estudian cadenas de suministro agrícolas a partir del uso de modelos matemáticos. En ¡Error! No se encuentra el origen de la referencia., se representan los conjuntos de palabras que se generan con los artículos consultados. La ilustración se construyó teniendo en cuenta una ocurrencia de cinco (5) palabras (se repite al menos cinco veces en los artículos consultados), lo que permitió identificar 16 palabras que representan temáticas específicas que se abordan al estudiar las cadenas de suministro agrícolas. 


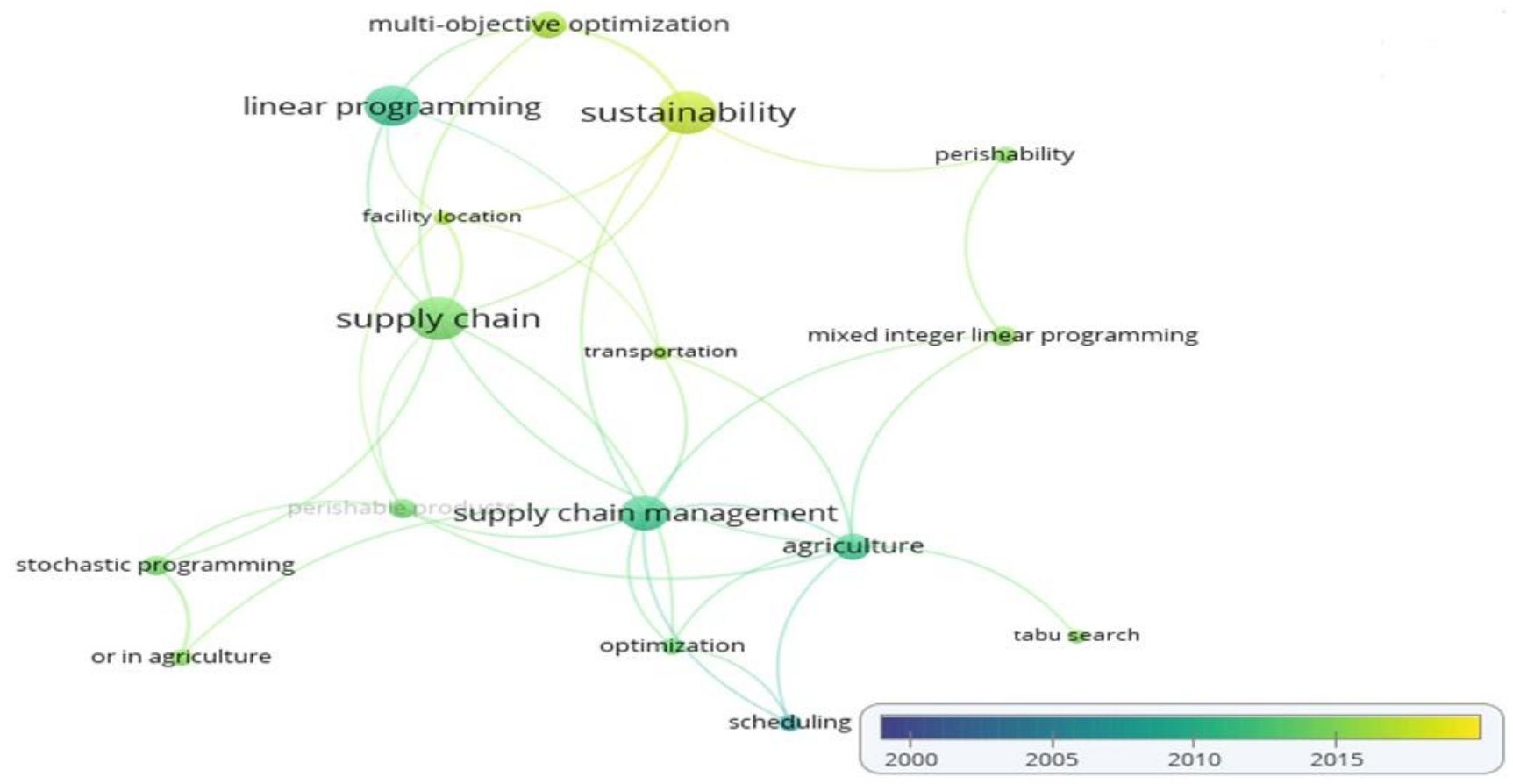

Ilustración 1. Agrupación de palabras clave de los artículos

Diversidad de autores abordan problemas sobre la gestión de la cadena de suministro, diseño de cadena a partir del análisis y ubicación de instalaciones, transporte de mercancías y el manejo de productos perecederos, entre otros temas importantes. Ha existido interés por abordar aspectos de sustentabilidad en las cadenas de suministro agrícolas, principalmente desde el año 2015 hasta la fecha.

Existe una relación directa entre la sustentabilidad y el desarrollo de modelos de optimización multiobjetivo. Esto se debe a que, para evaluar la sustentabilidad de los sistemas de abastecimiento, es necesario analizarla desde tres enfoques, el social, el ambiental y el económico. Diversidad de autores buscan evaluar simultáneamente estos tres enfoques en la cadena de suministro evidenciando la relación existente entre estos.

La Programación Lineal ha cobrado mayor relevancia frente a otros tipos de programación para la solución de modelos de optimización en agrocadenas, siguen siendo prácticas para la solución de problemáticas existentes las cadenas de suministro agrícolas; sin embargo, la Programación Lineal Entera Mixta y la Programación Estocástica (Stochastic programming), tienen una importante participación en el estudio de las agro-cadenas. Estos dos tipos de programación permiten abordar problemas más complejos, como la perecibilidad de alimentos y el ruteo de vehículos.

\section{RESULTADOS}

Se realiza el análisis a partir de la identificación de las tendencias de los tipos de modelos utilizados. Dependiendo del enfoque y tipo de producto de origen agrícola, los autores plantean metodologías, variables y modelos matemáticos de manera diferente, en los cuales algunas veces llegan a ser tan complejos, que es necesario de la utilización de la tecnología computacional para llegar a conseguir una solución óptima en tiempos relativamente cortos. Se identificó la tendencia, el tipo de estudio, aplicación de modelos matemáticos, algoritmos utilizados y las variables estudiadas entre otros subtemas de interés.

Los resultados de la tendencia de investigación presentan datos bibliográficos para contextualizar y descomponer los temas siguientes. Se realiza el análisis del contenido de 200 artículos y se sintetiza el estado del arte en estos elementos clave, como se muestra en los apartados 4.1 a 4.7 . 


\subsection{Tendencias de la investigación}

Inicialmente se realizó un análisis del año en que fueron publicados los diferentes artículos involucrados en el estudio, esto a partir del año 2000, con el fin de realizar una interpretación actualizada de los nuevos avances de investigación aplicados en agro cadenas.

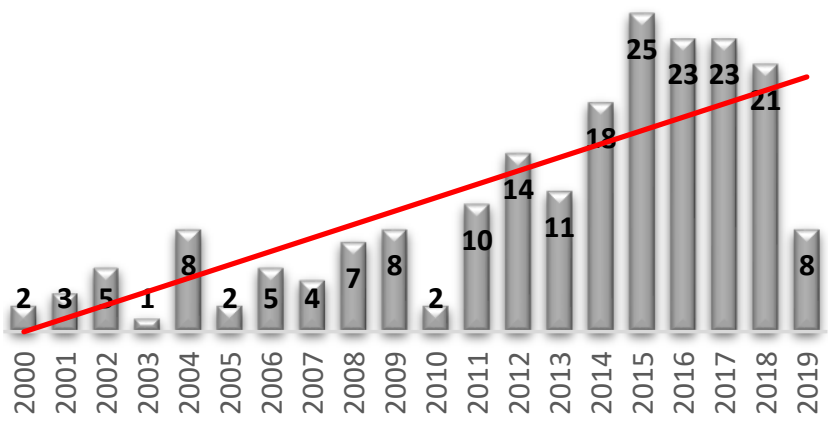

Año

Figura 1. Distribución de Artículos por Año.

Se visualiza en la ¡Error! No se encuentra el origen de la referencia., que el periodo comprendido entre el año 2000 hasta 2010 no superaron la cifra de 8 publicaciones anuales, es decir que se tuvo un promedio de 4 documentos en este periodo, lo que sugiere que en este lapso de tiempo existió poco interés por la comunidad científica para estudiar cadenas de suministro agrícolas. En el lapso de tiempo 2011 a 2018 se ve in incremento importante de publicaciones respecto al tema, contando con un mínimo de publicaciones de 10 en el 2011 y un máximo de 25 en el 2015. Esto indica que en este periodo existió un interés por la comunidad científica por el estudio de las Agrocadenas a partir del uso de modelos matemáticos. A partir del 2015, las publicaciones el número de publicaciones disminuyo, lo que sugiere que las temáticas siguen siendo relevantes, pero han llegado a su punto máximo de producción académica. Para el año 2019 se registran 8 documentos publicados, cifra que no se puede interpretar como una disminución, ya que, por el tiempo en el cual se realizó esta investigación no incluyó el total de meses del 2019, y es de esperarse que para el restante de 2019 se logren cifras cercanas a las de los anteriores 4 años. Es evidente que existe una tendencia creciente a la investigación en las agro-cadenas a partir de la modelación matemática, lo que sugiere que este sector de las economías se ha convertido en un blanco objetivo para comunidad científicoacadémica.

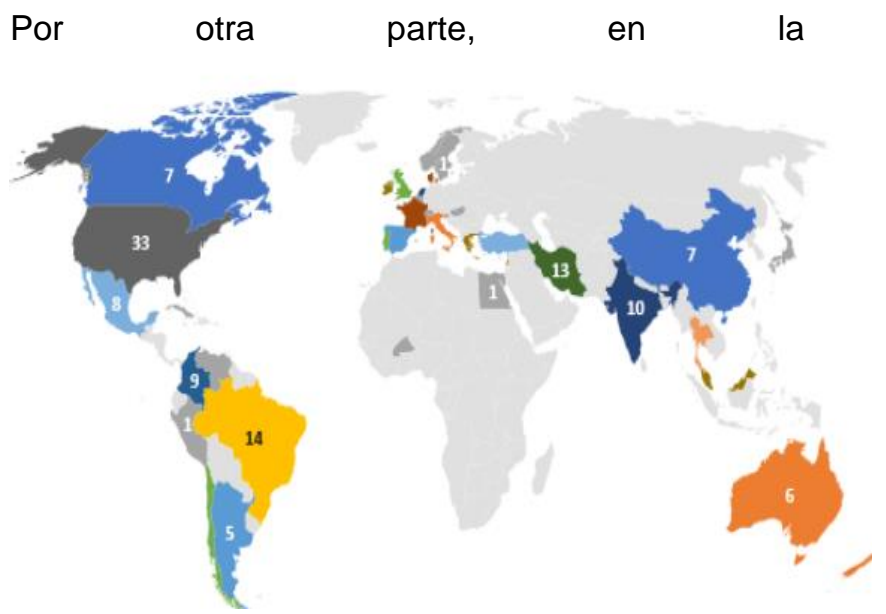

Ilustración 2 se muestra el número de publicaciones por país. El 60 \% (120 documentos) de los artículos analizados fueron aplicados de la siguiente manera; en Norteamérica se aplicaron un total de 32 investigaciones, donde el país dominante es Estados Unidos seguido de México; en Sudamérica se desarrollaron 25 investigaciones contando con la mayor parte en Brasil y Colombia; por otra parte en Europa se realizaron 31 investigaciones, país dominante Holanda y España; Asia con 24 artículos, país dominante Irán e India, 2 investigaciones en África y 6 investigaciones en Australia. Esto sugiere que el continente americano es pionero en el número de investigaciones sobre agro-cadenas a partir de la modelación matemática. Estados unidos es el país que mayor número de publicaciones ha realizado en los últimos 20 años, contando con un $16,5 \%$ de las publicaciones (33 documentos), seguido esta Brasil con un 7\% (14 documentos), después Irán con un 6,5\% (13 documentos), la India con un $5 \%$ (10 documentos), Holanda con un $5 \%$ (10 documentos) y Colombia con un 4,5\% (9 documentos) publicados. 


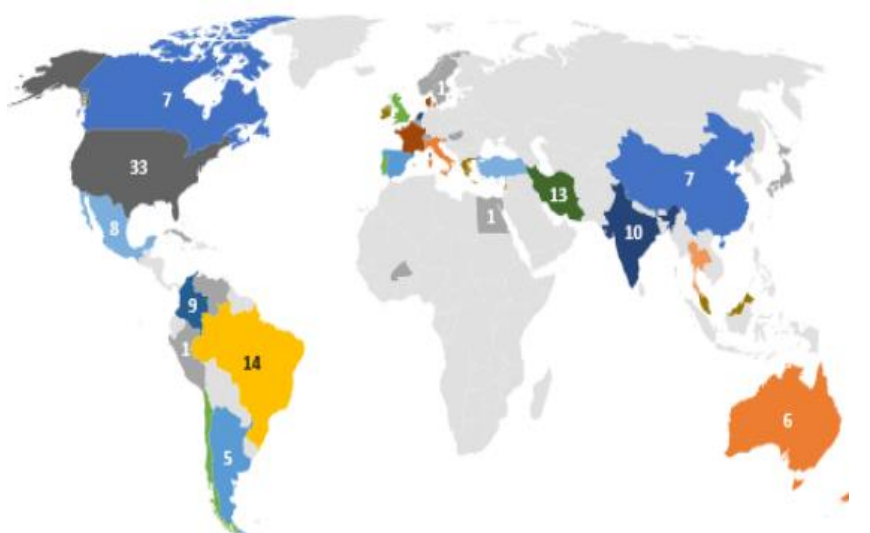

llustración 2. Países caso estudio

Las investigaciones que se han desarrollado se hacen principalmente en un ámbito nacional, esto para realizar abastecimiento a nivel interno en sus países. Entre algunos estudios que se desarrollan en estos países, se tiene el trabajo de Liu, Bai, \& Chen, [18], quienes desarrollan un marco de modelado de redes de transporte intermodal en estados unidos, centrado en los envíos de contenedores de soya; el objetivo fue evaluar el desempeño del transporte, identificar los cuellos de botella en la infraestructura y comparar las estrategias de inversión. En Irán se tiene el trabajo de Morales Chávez, Soto \& Sarache [19], quienes identifican que la gestión de logística es uno de los problemas más frecuentes a lo largo del año porque deben determinar las cantidades óptimas de trigo que se transportarán de cada provincia productora a cada provincia consumidora. Por otra parte, en Brasil se buscar adaptar los sistemas de producción agrícola, con el objetivo de obtener mejores rendimientos sin dejar de lado el manejo adecuado de los recursos naturales; ejemplo de ello es trabajo de Rocco \& Morabito [20], quienes presentan un problema de planeación de producción y la logística que enfrenta la industria brasileña en el procesamiento de tomate; Construyen un modelo de programación lineal para representar y apoyar adecuadamente la toma de decisiones en actividades agrícolas [20]. Por otro lado, en el trabajo de Morales Chávez, Sarache, \& Costa [21], proponen un modelo de MIP (programación lineal entera mixta) para el diseño de una cadena de suministro sustentable utilizando residuos generados en el cultivo del café; el modelo propone una estrategia de capacidad dinámica, que analiza sus efectos la ubicación de las instalaciones, el transporte y el flujo de materiales [21].

De los 200 documentos analizados, se encontró que 59 revistas estuvieron presentes en las publicaciones. Estas se visualizan en la Tabla 1.

Tabla 1. Distribución de artículos revisados por revista.

\begin{tabular}{lcc}
\hline \multicolumn{1}{c}{ Revista } & Artículos & Clasificación \\
\hline Agricultural Systems & 8 & Q3 \\
European Symposium on Computer Aided Process Engineering & 3 & Q3 \\
International Transactions in operational Research & 4 & Q2 \\
Energy Procedia & 1 & Q2 \\
Food Control & 1 & Q2 \\
No especifica & 1 & Q2 \\
Resources, Conservation and Recycling & 1 & Q2 \\
Journal of Chemical Technology and Metallurgy & 29 & Q1 \\
Computers and Electronics in Agriculture & 23 & Q1 \\
European Journal of Operational Research & 19 & Q1 \\
International Journal of Production Economics & 12 & Q1 \\
Computers and Industrial Engineering & 7 & Q1 \\
Applied Mathematical Modelling & 5 & Q1 \\
Biosystems Engineering & 5 & Q1 \\
Transportation Research & 4 & Q1 \\
Applied Energy & 4 & Q1 \\
Biomass and Bioenergy & 4 & Q1 \\
Journal of Food Engineering & 4 & Q1 \\
Journal of the Operational Research Society & 4 & Q1 \\
International Federation of Automatic Control & Q1 \\
Agricultural Water Management & 3 & Q1 \\
Applied Soft Computing & 3 & Q1
\end{tabular}




\begin{tabular}{lcc}
\hline \multicolumn{1}{c}{ Revista } & Artículos & Clasificación \\
\hline Annals of Operations Research & 2 & Q1 \\
Manufacturing \& Service Operations Management & 2 & Q1 \\
Renewable and Sustainable Energy Reviews & 2 & Q1 \\
Agricultural and Forest Meteorology & 1 & Q1 \\
Alexandria Engineering Journal & 1 & Q1 \\
American Institute of Chemical Engineers & 1 & Q1 \\
APCBEE Procedia & 1 & Q1 \\
Biomass Supply Chains for Bioenergy and Biorefining & 1 & Q1 \\
Conference on Intelligent Computation in Manufacturing Engineering & 1 & Q1 \\
Contaduria y administración & 1 & Q1 \\
Control Engineering Practice & 1 & Q1 \\
Engineering Applications of Artificial Intelligence & 1 & Q1 \\
Expert SystemsWith Applications & 1 & Q1 \\
Fisheries Research & 1 & Q1 \\
Food Policy & 1 & Q1 \\
Industrial Aplications & 1 & Q1 \\
Industrial Crops \& Products & 1 & Q1 \\
International Journal of Systems Science & 1 & Q1 \\
Journal of Integrative Agriculture & 1 & Q1 \\
Journal of Ocean Engineering and Science & 1 & Q1 \\
Livestock Science & 1 & Q1 \\
OR Spectrum & 1 & Q1 \\
Production and Operations Management Society & 1 & Q1 \\
Research in Transportation Business \& Management & 1 & Q1 \\
Scientia Horticulturae & 1 & Q1 \\
Journal of Cleaner Production & 6 & N/A \\
Computers and Operations Research & N/A \\
Energy & N/A \\
Computers and Chemical Engineering & N/A \\
The international journal of management science & N/A \\
Computers in Industry & N/A \\
Forest Policy and Economics & 2 & N/A \\
Journal of Manufacturing Systems & 2 & N/A \\
Journal of Transport Geography & 2 & N/A \\
Robotics and Computer-Integrated Manufacturing & 1 & N/A \\
\hline Totainable Production and Consumption & 1 &
\end{tabular}

Se identifican cuatro revistas que representan cerca del $42 \%$ de los artículos publicados: Computers and Electronics in Agriculture con 29 documentos $(14,5 \%)$, European Journal of Operational Research con 23 documentos (11,5\%), International Journal of Production Economics con 19 documentos (9,5\%) y Computers and Industrial Engineering con 12 documentos (6,0\%). Esto indica que estas cuatro revistas tienen gran visibilidad e impacto en la comunidad científica.

De igual forma, la clasificación de estas revistas categorizándolas en Q1, Q2, Q3 Y Q4 certifica que un porcentaje importante de trabajos fueron sometidas en revistas de alto impacto. Esto soporta el argumento de que el estudio de cadenas de suministro agrícolas a partir de la modelación matemática ha tenido un impacto importante en la comunidad científica durante los últimos 20 años.

\subsection{Tipo de estudio y alcance}

En la ¡Error! No se encuentra el origen de la referencia. , se muestra el alcance que tuvieron los 200 estudios involucrados en la revisión. Se categorizó en un alcance estratégico, táctico, operativo y algunas combinaciones de estos. 


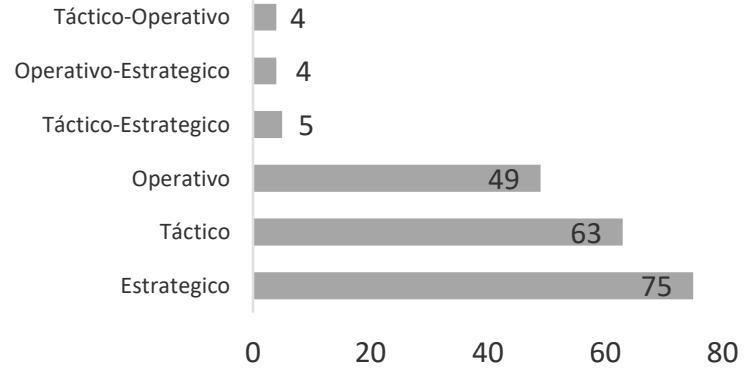

Figura 2. Alcance de la investigación.

La mayor cantidad de trabajos buscan la solución de problemas de orden estratégico y táctico, lo cual lleva a pensar que, en los últimos 20 años ha sido vital la planificación a largo y mediano plazo en las agro-cadenas. Esto sin dejar de lado la solución de problemas de orden operativo dado que un número importante de trabajos buscan solucionar problemas de este tipo.

\subsection{Métodos de solución de modelos}

Actualmente, el uso de modelos matemáticos se ha incorporado para facilitar la toma de decisiones y es aplicado en diversos sectores con resultados muy satisfactorios. Algunos ejemplos de sus usos en la agricultura son: modelos para la producción de bienes, modelos para determinar el rendimiento de los sistemas logísticos, modelos de ruteo de vehículos, entre otros.

La Tabla 2 muestra los tipos de modelos empleados por los 200 autores, clasificándolos en estocásticos y determinísticos.

Tabla 2. Tipo de modelo.

\begin{tabular}{ccc}
\hline Tipo de Modelo & Cantidad & $\%$ \\
\hline Determinístico & 128 & $64 \%$ \\
Estocástico & 72 & $36 \%$ \\
\hline Total & $\mathbf{2 0 0}$ & $\mathbf{1 0 0 \%}$ \\
\hline
\end{tabular}

No se establece una premisa sobre cual método es mejor, simplemente se muestra el panorama sobre cual se ha usado más dado que, el método que se emplee dependerá de las características de la problemática específica que se esté abordando en cada agro cadena. El $36 \%$ de los artículos analizados corresponde a modelos de simulación estocástica; se evidencia que, en los últimos 20 años en la comunidad científica, ha sido relevante el uso de este tipo de modelos para la solución de problemas complejos, de igual estos modelos permiten abordar problemas con altos grados de incertidumbre de alto nivel. Esto pude estar ocasionado por las facilidades tecnológicas que permiten el uso de estos modelos en tiempos relativamente cortos, simplemente el grado de detalle bajo el cual se desea estudiar la agrocadena. En el $64 \%$ de los estudios emplean modelos de tipo determinístico para abordar los problemas asociados al estudio de las agrocadenas. Los modelos determinísticos son aplicados en situaciones reales donde su comportamiento está alineado a las características iniciales.

Por otra parte, los modelos matemáticos pueden clasificarse según el enfoque de solución: puede ser heurística o meta heurística. Se identifica un amplio margen de diferencia entre los dos enfoques de solución, siendo la heurística la de mayor presencia con 141 documentos analizados lo que representa el $70 \%$ del total de artículos. Entre estos se destaca el trabajo de Etemadnia, Goetz, Canning, \& Tavallali [22], en donde se diseña una red óptima de ubicación nacional para proveer a los mercados de consumo de alimentos mediante la formulación de un modelo MIP que minimiza los costos de la red, consiguiendo con esta heurística una reducción considerable del tiempo de cálculo. En cuando al enfoque de solución Meta-Heurístico se obtuvo que un $30 \%$ de los documentos revisados pertenecen a esta categoría. La complejidad de algunos problemas hace que no puedan ser solucionados por métodos heurísticos [23], para solucionarlos en un tiempo razonable se requieren métodos metaheurísticos que resultan ser más eficientes que los métodos exactos; sin embargo, el enfoque de solución más allá de ser una elección de los autores se puede convertir en una obligación, esto dependiendo de la complejidad del modelo planteado.

Hoy día, con los tipos de programación matemática, es posible integrar toda la cadena de suministros, para optimizar y mejorar su rendimiento total en todos sus eslabones. En la Figura 3 se presentan los tipos de programación que se emplean en los 200 trabajos consultados. 


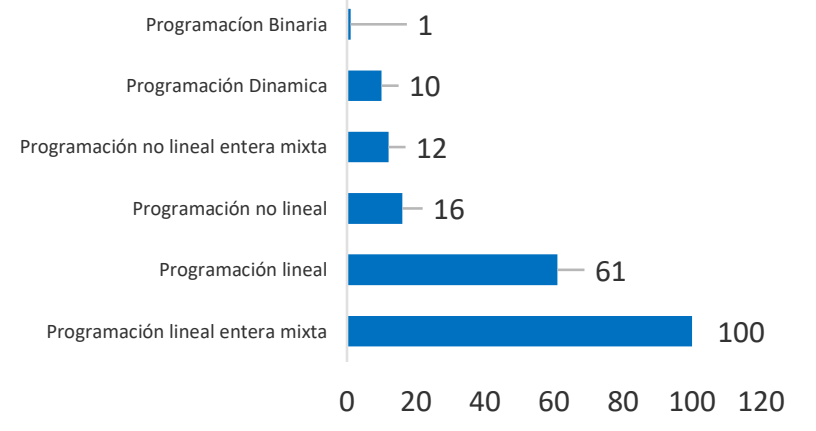

Figura 3. Tipo de programación.

De acuerdo con la figura, los modelos MIP (50\%) son los que más se emplean para el estudio de agrocadenas. Seguido a ello se encuentra los modelos LP (Programación Lineal) con 31\% y los de NLP (programación no lineal), $8 \%$. Esto sugiere que, para el estudio de la agro-cadenas la programación entera mixta se convierte en una herramienta fundamental para la solución de modelos matemáticos que representan los sistemas agrícolas.

Los modelos de MIP, LP y NLP, son ampliamente utilizados para la solución de problemas complejos en todos los niveles organizacionales, permiten alcanzar una reducción de costos operativos, un incremento productividad [24], el aprovechamiento de capacidad y el control de inventarios [25]. Así mismo, periten el uso eficiente de recursos, cumplir con requerimientos de clientes y lograr un desarrollo sostenible [26].

Las empresas que experimentan un rápido crecimiento, enfrentan una amplia gama de oportunidades para reducir costos productivos y logísticos. Ejemplo de ello es el trabajo de Junqueira \& Morabito [15], donde estudian la aplicación de un modelo LP para respaldar las decisiones en la planeación del almacenamiento, el transporte y la producción de semillas de maíz en toda una temporada de una empresa brasileña típica de semillas de múltiples plantas. En el trabajo de Springael, Paternoster, \& Braet [27], se evaluó un método no lineal eficaz para reducir la tasa de pérdida de manzanas y minimizar el número y la intensidad de las vibraciones que se producen durante la ruta de transporte. Por otro lado, Mogale, Krishna, \& Kumar [28], desarrollaron un modelo MINLP (Programación No Lineal Entera Mixta) para planificar el movimiento y almacenamiento de granos alimenticios desde estados con superávit a estados deficitarios, considerando las adquisiciones estacionales, la capacidad del silo, la satisfacción de la demanda y las limitaciones de capacidad del vehículo. La programación binaria es la que menos se emplea en la literatura como lo hacen autores como Tordecilla et al [29], quienes buscan la localización de tanques de enfriamiento de leche en un municipio de Cundinamarca - Colombia.

\subsection{Algoritmos de solución}

En la Tabla 3 se presentan los algoritmos utilizados para dar solución a diferentes problemas asociados al estudio de las agro-cadenas, así como el enfoque de aplicación y los autores que los abordan.

Tabla 3. Algoritmos.

\begin{tabular}{llcl}
\hline Autores & \multicolumn{1}{c}{ Algoritmo } & Cantidad & \multicolumn{1}{c}{ Aplicación } \\
\hline [30]; [31]; [32]; [33]; [34]; [35]; [36]; [37] & Algoritmo genético (GA) & 8 & Distribución \\
[38]; [22] [39]; [40]; [41]; [42]; [43] & Algoritmo Branch-and-cut & 7 & Incertidumbre \\
[44]; [23]; [45] [25] & Partículas de enjambres múltiples (PSO) & 4 & Distribución \\
[46]; [47]; [48] & Algoritmo de simulación de Recocido (SA) & 3 & Temperatura \\
[49] [50] & Algoritmo de descomposición de Bender & 2 & Planificación \\
[51] & Algoritmo de ahorro Clarke-Wright & 1 & Ruteo \\
{$[52]$} & Algoritmo de barrera & 1 & Planificación \\
[53] & Algoritmo de generación de columnas & 1 & Producción \\
[54] & Algoritmo de horizonte rodante & 1 & Incertidumbre \\
& & & (Demanda) \\
[55] & Algoritmo de optimización de reacción química & 1 & Distribución
\end{tabular}


[28]

[59]

\begin{tabular}{lll}
$\begin{array}{l}\text { Algoritmo de optimización de reacción química } \\
\text { de partículas híbridas (HP-CRO) }\end{array}$ & 1 & Distribución \\
$\begin{array}{l}\text { Algoritmo de programación dinámica (HDPA) } \\
\text { Algoritmo de Schmenner }\end{array}$ & 1 & $\begin{array}{l}\text { Distribución } \\
\text { Producción } \\
\text { Aistribución }\end{array}$ \\
$\begin{array}{l}\text { Algoritmo Keshtel (MOKA) de objetivos } \\
\text { múltiples }\end{array}$ & 1 & $\begin{array}{l}\text { Incertidumbre } \\
\text { Muestreo dinámico de aproximación externa }\end{array}$ \\
$\begin{array}{l}\text { Algoritmo (DOASA) } \\
\text { Total }\end{array}$ & 1 & 34 \\
\hline
\end{tabular}

De los 200 trabajos consultados, 34 emplean algún tipo de algoritmo para la solución de diversidad de problemas asociados a las Agro-cadenas. Los algoritmos genéticos (GA) son los que más se utilizan, principalmente se emplean para la solución de problemas asociados a la distribución o abastecimiento de mercancías a lo largo de las agro-cadenas. De igual forma, algoritmos como Partículas de enjambres múltiples (PSO), de optimización de reacción química (CRO), optimización de reacción química de partículas híbridas (HP-CRO), de programación dinámica (HDPA) y de Algoritmo Keshtel (MOKA) de objetivos múltiples han permitido abordar este tipo de problemas. Los algoritmos de Branch-and-Cut ([38]; [22] [39]; [40]; [41]; [42]; [43]), Algoritmo de horizonte rodante ([54]) y el de Muestreo dinámico de aproximación externa Algoritmo ([59]) han sido usados para la solución de problemas con gran grado de incertidumbre en el comportamiento de las variables de rendimiento de la cadena, principalmente en la medición de la demanda. Para la solución de problemas de planificación en agrocadenas o aspectos relacionados con la producción de productos agrícolas, se han empleado algoritmos como el de descomposición de Bender ([49] [50]),
Algoritmo de barrera [52], Algoritmo de Schmenner [57] entre otros. Esto evidencia que, los problemas presentados en las agro-cadenas pueden ser de gran complejidad, por lo que demandan el desarrollo de algoritmos que permitan la solución de modelos que represente la complejidad de estos sistemas.

\subsection{Variables estudiadas}

En la

Tabla 4 se presenta las variables que se han abordado para la medición y mejora del rendimiento de las Agro-cadenas. Tambien se muestra el número de funciones objetivos que contempló cada autor para aumentar el rendimiento de la cadena. Cabe resaltar que, en los 200 artículos consultados, diversidad de autores buscan el mejoramiento de diversas variables, como la robustez, la resiliencia, el impacto ambiental, los costos la calidad, los costos entre otros; sin embargo, esto no implica que necesariamente todas variables la mida directamente con el cálculo de la función objetivo, muchos autores abordan diferentes variables con su análisis e interpretación a partir de la información paramétrica empleada y el usos de restricciones dentro de los modelos.

Tabla 4. Variables utilizadas y función objetivo.

\begin{tabular}{|c|c|c|c|c|c|c|c|c|c|c|c|c|c|c|c|c|}
\hline Autores & \# FO & 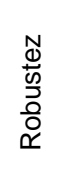 & 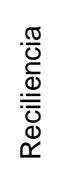 & 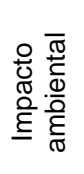 & 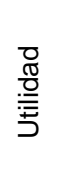 & $\begin{array}{l}\text { ô } \\
\text { in } \\
\text { Oे }\end{array}$ & $\begin{array}{l}\frac{\bar{d}}{\mathbb{D}} \\
\frac{\overline{0}}{\bar{J}} \\
0\end{array}$ & 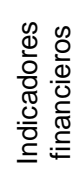 & $\begin{array}{l}\frac{0}{0} \\
\frac{0}{\mathrm{U}} \\
\frac{\mathrm{O}}{\overline{0}} \\
\overline{0} \\
\overline{0} \\
0 \\
0\end{array}$ & 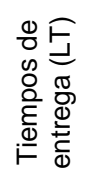 & 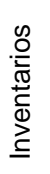 & $\frac{\overline{\frac{\sigma}{\pi}}}{\stackrel{5}{\nu}}$ & 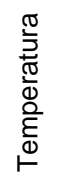 & 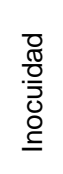 & 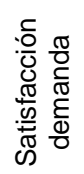 & $\begin{array}{l}\mathscr{0} \\
\widetilde{\Xi}\end{array}$ \\
\hline [60] & Uno & & & $X$ & & & & & $X$ & & $X$ & & & & & \\
\hline [2] & Tres & & & $\hat{x}$ & $x$ & $x$ & & & & & & & & & & \\
\hline [5] & Uno & & & $\mathrm{X}$ & & & $x$ & $x$ & & & & & & & & \\
\hline$[9]$ & Tres & & & $x$ & & & $x$ & & & & & & & & & $x$ \\
\hline [16] & Uno & & & $\hat{x}$ & & $x$ & & & $x$ & & & & & & & \\
\hline [14] & Dos & & & & & & & $x$ & & & & & & & & $x$ \\
\hline [15] & Uno & & & & & $x$ & & & & & & & & & & $x$ \\
\hline [4] & Uno & & & & & $\hat{x}$ & $x$ & & & & & & $x$ & & & \\
\hline [8] & Uno & & & & & $x$ & & & & & & & & & & $x$ \\
\hline [18] & Uno & & & & & $\hat{x}$ & & $x$ & & & & & & & & $\hat{x}$ \\
\hline
\end{tabular}




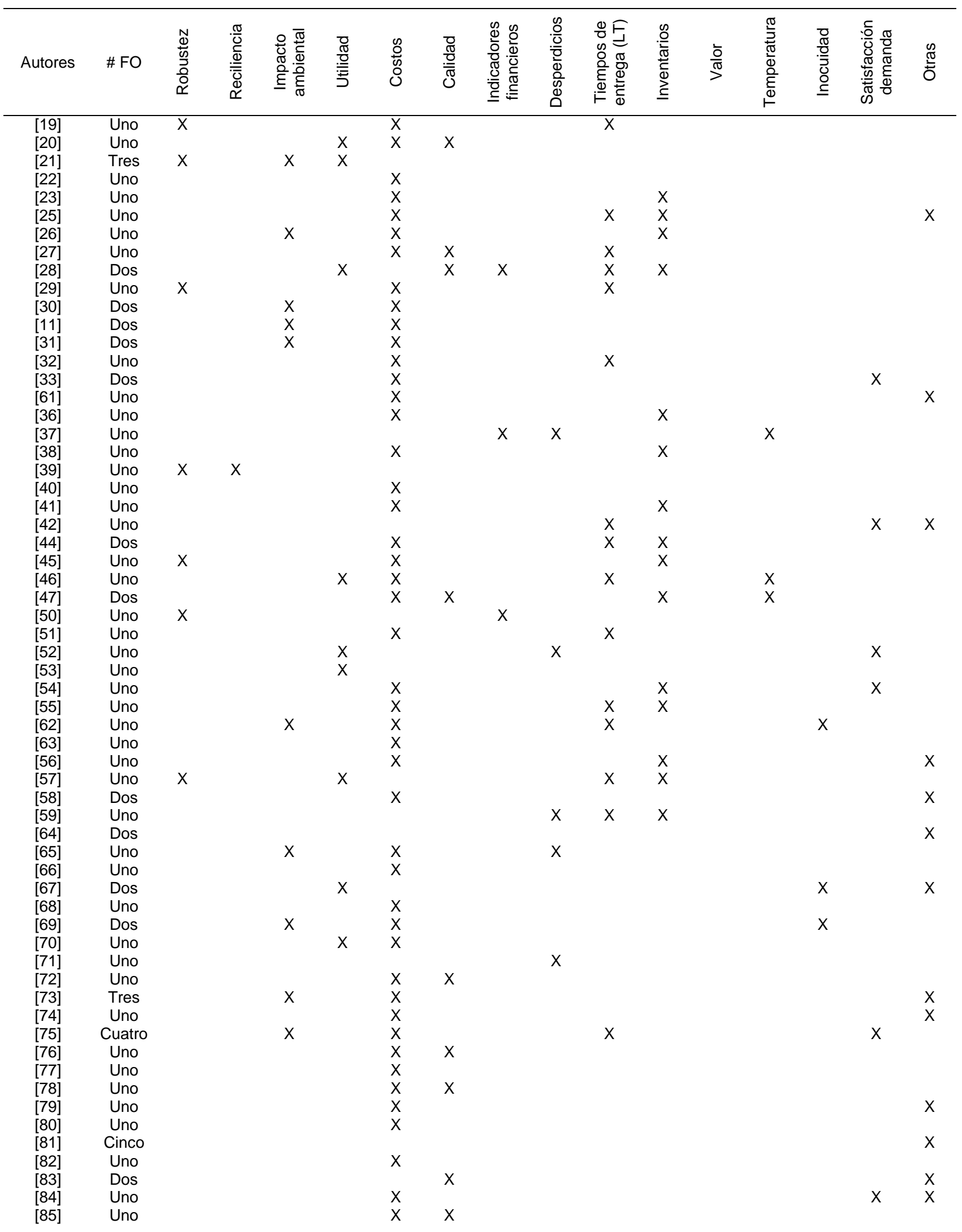




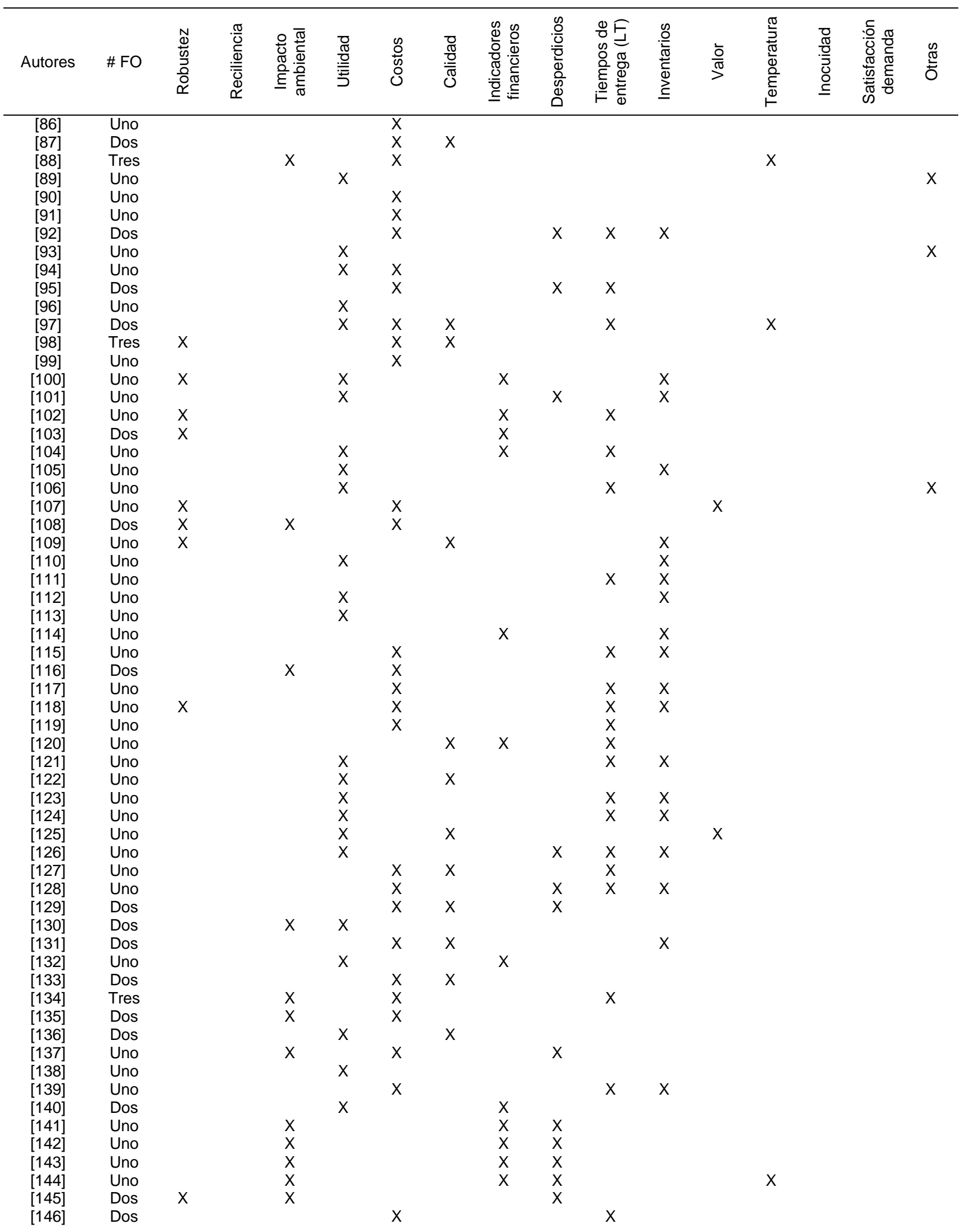




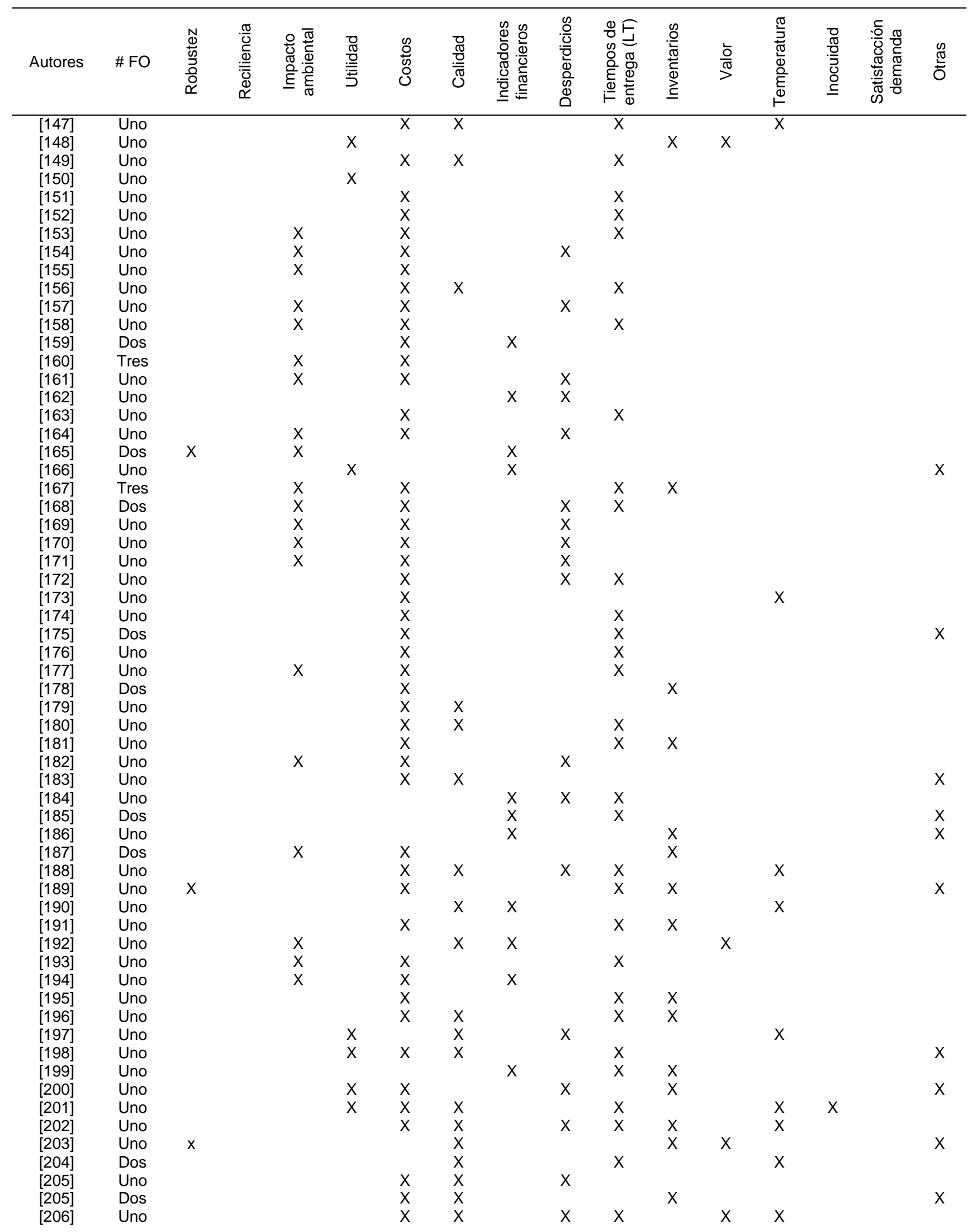




\begin{tabular}{|c|c|c|c|c|c|c|c|c|c|c|c|c|c|c|c|c|}
\hline Autores & \# FO & 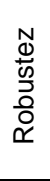 & 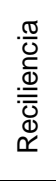 & 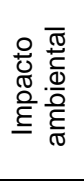 & 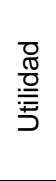 & 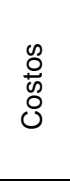 & $\begin{array}{l}\frac{\bar{\pi}}{\mathbb{\pi}} \\
\frac{.0}{\bar{T}} \\
0 \\
0\end{array}$ & 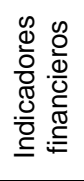 & $\begin{array}{l}\stackrel{0}{0} \\
\frac{0}{0} \\
\frac{0}{0} \\
\overline{0} \\
\frac{0}{0} \\
0 \\
0\end{array}$ & 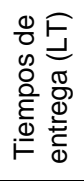 & 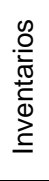 & $\frac{\frac{\overline{0}}{\pi}}{\frac{\pi}{\nu}}$ & 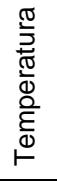 & 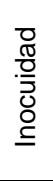 & 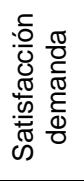 & 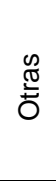 \\
\hline [207] & Dos & & & $\mathrm{X}$ & & $\mathrm{X}$ & $\mathrm{X}$ & & $x$ & & & & & & & $x$ \\
\hline [208] & Uno & & & & & & $X$ & $X$ & & X & $X$ & & $x$ & & & \\
\hline [209] & Uno & $X$ & & & & $X$ & $X$ & & & & $x$ & & & & & \\
\hline [210] & Uno & $X$ & & $X$ & & X & $x$ & & & & & & $x$ & & & \\
\hline [211] & Dos & & & $x$ & & $x$ & & & & $x$ & & & & $x$ & & $x$ \\
\hline [212] & Uno & $x$ & & & & $x$ & & $x$ & & & & & & & & \\
\hline [213] & Uno & $\mathrm{X}$ & & & & X & & & & X & $X$ & & & & & $X$ \\
\hline & & 23 & 1 & 50 & 41 & 133 & 47 & 31 & 38 & 70 & 53 & 6 & 18 & 5 & 6 & 34 \\
\hline
\end{tabular}

Las variables que más se analizan por la comunidad científica, se tiene los costos (133 trabajos), el lead time o tiempo de entrega (70 trabajos), el impacto ambiental (50 trabajos), calidad (47) y la utilidad (41 trabajos) entre otros. Esto muestra que, varios autores se centran por mejorar el rendimiento de la cadena a partir la medición de variables como los costos y los tiempos de respuesta; sin embargo, esto no quiere decir que no presten atención a temas asociados a sostenibilidad ambiental, dado que un número importante de investigaciones han abordado este aspecto. El estudio de impactos ambientales de la operación de las agro-cadenas ha cobrado un interés importante por la comunidad científica. Por otra parte, un total de 70 estudios buscan abordar temas relacionados con la calidad (47 trabajos), control de la temperatura (18 trabajos) e innocuidad (5 trabajos), lo que indica que, en los últimos 20 años el cuidado y preservación de los productos agrícolas ha sido un factor importante que tener en cuenta al momento de estudiar las cadenas de suministro. Por otra parte, en 24 estudios se busca mejorar la robustez (23 trabajos) y la resiliencia (1 trabajo [39]) de las cadenas de suministro agrícolas, lo que representa que ha cobrado importancia el análisis y generación de estrategias para asegurar que las cadenas de suministro sean los suficientemente estables operar en escenarios de incertidumbre.

Por lo general, los modelos matemáticos abordan máximo dos funciones por el grado de complejidad que conlleva la solución de estos; sin embargo, hay autores que innovan con la formulación más de tres funciones objetivos. En las investigaciones realizadas por Mohammed \& Wang [75], y Biswas \& Baran [81], se trabajan cuatro y cinco funciones objetivos respectivamente. En el trabajo de Mohammed \& Wang [75], se propone un modelo matemático con cuatro funciones objetivo que buscan minimizar el costo total de transporte e interpretación, minimizar la cantidad de emisiones de $\mathrm{CO} 2$ en el transporte, minimizar el tiempo de distribución de los productos y maximizar la tasa de entrega promedio para satisfacer la demanda del cliente en una cadena de suministro de carne de res; para lo cual, fue necesario utilizar tres métodos de solución como métricas de programación lineal, método de restricción y programación por objetivos logrando determinar el número de instalaciones con ubicaciones que deben abrirse con un equilibrio entre las cuatro funciones objetivas propuestas; En el trabajo de Biswas \& Baran [81] se formula una quinta función objetivo, haciendo que ese sea el único documentos con tal número de funciones y que a diferencia de otros documentos no incluye objetivos ambientales 0 sociales, pues sus funciones objetivo buscan optimizar la utilización de la tierra, la producción, recursos para la producción, gasto de dinero y finalmente el beneficio económico.

\subsection{Sector de estudio}

Para el análisis se categorizaron los artículos consultados de acuerdo al subsector (agricultura, ganadería, silvicultura, apicultura y minería), como se puede ver en la ilustración Figura 4. Distribución de artículos revisados por sector. 


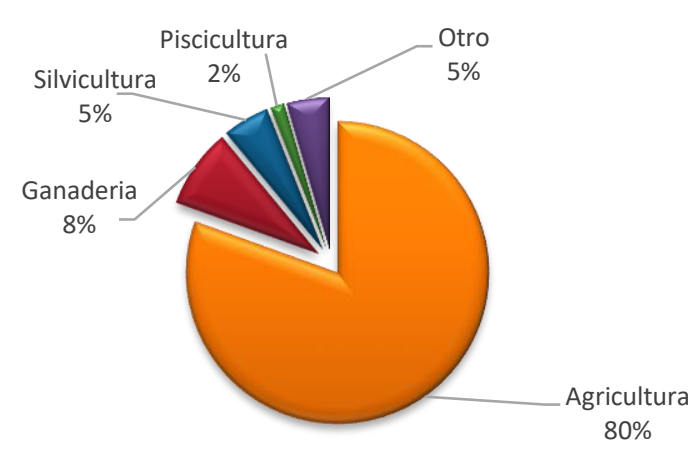

Figura 4. Distribución de artículos revisados por sector.

En

Figura 4 se observa que el mayor porcentaje de las investigaciones se centra en el sector de la agricultura con un $80 \%$ (159 documentos), lo que era de esperarse dado al enfoque de la presente investigación; sin embargo, el $8 \%$ fueron realizados en el subsector de ganadería (17 documentos), el 5 $\%$ en silvicultura (10 documentos), el $2 \%$ en piscicultura (3 documentos), y el $5 \%$ correspondientes a otros sectores (9 documentos), esto muestra que, existen agro-cadenas en estos subsectores que requieren apoyo de la comunidad científica. De igual forma, que estos subsectores no han sido muy explorados desde un punto de vista científico, por tanto, tienen un potencial en generación de nuevo conocimiento.

En la agricultura se abordan temas de calidad, costos, logística, desperdicios, medio ambiente entre otros para el abastecimiento de frutas, verduras, cereales, flores y biocombustibles a partir de residuos agrícolas abordan; sin embargo, el sector agrícola todavía carece de herramientas y modelos para mejorar la utilización de diferentes recursos pese a los importante avances e investigación en el sector. Cada estudio de los encontrados se enfoca en una problemática particular, y existe diversidad de problemáticas relacionadas con el estudio de agro-cadenas. Ejemplo de ellos es el trabajo de Arnaout \& Maatoukw [43], quienes abordan el problema de la cosecha de viñedos en los países en vía de desarrollo, con buscando determinar el mejor programa de cosecha que minimice los costos de las cosechadoras, así como las pérdidas de calidad de las uvas; Así mismo el trabajo de Blackburn \& Scudder [206], quienes examinan las estrategias de diseño de la cadena para un tipo específico de producto perecedero, usando melones y maíz dulce; En el estudio de Paulo, Azcue, Barbosa, \& Relvas [173], plantean la idea que la bioenergía puede representar una solución atractiva de energía renovable si se administra de manera efectiva.

En el subsector de la ganadería se encuentran trabajos como el de Pia, \& Alonso [177], quienes presentan presenta un modelo de planeación de producción para gestionar el sistema de producción de hatos de porcinos como una Cadena de Alimentación de Cerdo (PSC). En la piscicultura se abordan temas de planificación y distribución buscando mantener la cadena de frio, esto involucrando el análisis de costos, la calidad e impactos ambientales, ejemplo de ello es el trabajo de Pathumnakul, Piewthongngamb, \& Khamjanc [91], quienes examinan el problema de coordinación en la cadena del camarón tailandés; de igual forma el trabajo de Bakhrankova \& Kjetil [113], o el de Tabrizi, Hassan \& Ahmadi [125], quienes abordan problemas de planeación y modelado de la cadena de piscícola.

\subsection{Herramientas tecnológicas para el estudio de agro-cadenas}

El uso de herramientas tecnológicas permite la consecución de resultados de forma rápida y precisa, por ello, en algunos estudios fue necesario el apoyo en la tecnología para la consecución de información. El 17\% de las investigaciones (35 trabajos) hacen uso de herramientas tecnológicas para la obtención de información para el estudio de las agro-cadenas (Tabla 5).

Tabla 5. Uso de herramienta tecnológica

\begin{tabular}{|c|c|c|c|}
\hline Herramienta & Cantidad & Costos & Tiempos de Entrega \\
\hline GPS & 12 & & $\mathrm{x}$ \\
\hline GIS (sistema de información geográfica) & 5 & & $x$ \\
\hline RFID & 3 & & $x$ \\
\hline Cartografía & 2 & & $\mathrm{x}$ \\
\hline
\end{tabular}


Google Maps

MySQL, HTML, CSS, JavaScript y PHP

Asten Plus

GPS, GoPro Hero y Acelerómetro

GPS: Receptores DGPS AgGPS 162

Interfaz actividad aérea

NIWA CliFlo

NTAD, BTS, bases de datos NDC, NETPAS y GIS

Satélite (SIMS), GIS-Optice

Sofware Simapro

Sugarmax

Visual Basic

\section{Total general}

La mayor parte de las herramientas tecnológicas se emplean para la recolección de información de tipo geográfico, principalmente para la solución de problemas de producción, localización y distribución de mercancía [30], [52], [75], [79], [100], [29], [121], [124], [133], [134], [137], entre otros, y problemas de ruteo de vehículos (VRP) y transporte [31], [32], [97], [27], [163], [33], [18], entre otros. Esto empleando principalmente herramientas como GPS, sistemas de información geográfica e identificación por radiofrecuencia (RFID) entre otras, que han permitido a diversidad de autores recolectar información paramétrica necesaria para el estudio de las agro-cadenas de forma práctica. De igual forma, los problemas que requieren el uso de algún instrumento de este tipo, buscan la mejora de variables como los costos o los tiempos de entrega, lo cual es coherente cuando se abordan problemas de distribución, localización y ruteo ve vehículos. Se evidencia que durante los últimos 20 años ha sido prescindible el uso de este tipo de tecnologías para el estudio y mejoramiento de las agro-cadenas.

Por último, una parte fundamental de todo modelo matemático son las herramientas computacionales requeridas para resolverlos. Dada la complejidad de algunos modelos, es necesario el desarrollo de software usado como herramientas de solución en tiempos relativamente cortos.

En la Tabla 6 se puede visualizar el software que se emplea para la solución de los modelos matemáticos en los 200 artículos consultados.

Tabla 6. Programa computacional. 


\begin{tabular}{lcc}
\hline \multicolumn{1}{c}{ Descripción } & \# Documentos & $\%$ \\
\hline CPLEX & 35 & $18 \%$ \\
SI PERO NO ESPECIFICA & 34 & $17 \%$ \\
MATLAB & 23 & $12 \%$ \\
GAMS Y CPLEX & 21 & $11 \%$ \\
GAMS & 20 & $10 \%$ \\
LINGO & 19 & $10 \%$ \\
EXCEL & 5 & $3 \%$ \\
CPLEX Y AMPL & 3 & $2 \%$ \\
GUROBI OPTIMIZER & 3 & $2 \%$ \\
NO UTILIZA & 3 & $2 \%$ \\
XPRESS-IVE & 3 & $2 \%$ \\
CPLEX Y OPL & 2 & $1 \%$ \\
EXCEL Y GAMS & 2 & $1 \%$ \\
MAPLE & 2 & $1 \%$ \\
AIMMS & 1 & $1 \%$ \\
CPLEX Y EXCEL & 1 & $1 \%$ \\
CPLEX Y GUROBI & 1 & $1 \%$ \\
OPTIMIZER & $1 \%$ \\
CPLEX Y MATLAB & $1 \%$ \\
CPLEX, LINGO Y EXCEL & 1 & $1 \%$ \\
DOASA & 1 & $1 \%$ \\
DSSAT Y APSIM & 1 & $1 \%$ \\
DYNAMIC DSP MODEL & 1 & $1 \%$ \\
EASYLOCAL ++ & 1 & $1 \%$ \\
EXCEL Y LINDO & $1 \%$ \\
FDP & $1 \%$ \\
FICO XPRESS MOSEL & $1 \%$ \\
GAMS Y PATH & $1 \%$ \\
GIS & 1 & $1 \%$ \\
GLPK Y AMPL & $1 \%$ \\
GMN Y MATLAB & 1 & $1 \%$ \\
GUROBI OPTIMIZER Y & $1 \%$ \\
MPL 4.2 & $1 \%$ \\
MATLAB Y GAMS & $1 \%$ \\
MOGA II, NSGA II E & $1 \%$ \\
HYBRID & $1 \%$ \\
MOSEL-XPRESS-IVE & $1 \%$ \\
PULP & $1 \%$ \\
STELLA & $1 \%$ \\
VENSIM PLE & $1 \%$ \\
XPRESS - MP & XPRESS-MOSEL & $1 \%$ \\
\hline TOtal & $1 \%$ & $1 \%$ \\
\hline
\end{tabular}

CPLEX es la herramienta de mayor uso para la solución de modelos matemáticos, esto se puede deber al tipo de programación requerida que, en su mayor parte son MIP y LP. Esto no quiere decir que, este software no permite otros tipos de programación o que, no se empleen otros softwares de optimización que empleen estas dos programaciones. Otras herramientas como MATLAB, GAMS y LINGO, también sus usados en gran número de investigaciones; estos permiten diversos tipos de programación y el dominio de grandes volúmenes de información, lo que es convenientes para la solución de problemas complejos de las Agro-cadenas. Adicionalmente, es importante resaltar que en el $98 \%$ de los trabajos consultados se manifiesta que se empleó una herramienta especifica de optimización, lo que muestra la importancia el desarrollo de softwares para la solución de modelos matemáticos.

\section{CONCLUSIONES Y RECOMENDACIONES}

La publicación de artículos enfocados en el estudio de las agro-cadenas a partir del uso de modelos matemáticos no fue muy alta durante el periodo 2000 a 2010; sin embargo, a partir del año 2011 hasta el 2019, múltiples autores han profundizado más en este campo de estudio, evidenciando una tendencia creciente de publicaciones sobre este tema. Esto indica que existe un interés bastante grande por la comunidad académico-científica por fortalecer el sector agrícola a nivel internacional y nacional. En diversidad de países se desarrollan estudios para solucionar problemas asociados a la cadena agrícola, entre los países que más se destacan, se encuentra Estados Unidos, Brasil, Irán, India y Colombia.

Por otra parte, en cuanto a las revistas en las que fueron publicados los artículos se encontró que, existen cuatro revistas que contienen el $41,5 \%$ de trabajos consultados, la Computers and Electronics in Agriculture, la European Journal of Operational Research, la International Journal of Production Economics y la Computers and Industrial Engineering, las cuales son revistas de alto impacto categorizadas en scimago journal rankings como Q1. Esto sugiere que, la producción académica ha contribuido con la generación de nuevo conocimiento de alta calidad sobre la temática de estudio.

En cuanto al alcance de los estudios consultados, se tienen el $37.5 \%$ buscan solucionar problemáticas a nivel estratégico, el $31.5 \%$ a nivel táctico, el $24.5 \%$ de orden operativo y el $6.5 \%$ restante buscan solucionar problemas en los tres niveles. Esto indica que, los modelos matemáticos son de utilidad tomar decisiones operativas, tácticas y estratégicas. Adicionalmente, el $61 \%$ de los estudios consultados se desarrollaron a partir de información real y/o aplicaron metodologías directamente en las agrocadenas impactando positivamente su desempeño. Lo que muestra que los avances en investigación no son simplemente teorías sin aplicación alguna, sino que han permitido solucionar diversidad de problemas presentes en las cadenas de suministro agrícolas. 
Se discrimina que el $36 \%$ de los artículos analizados corresponde a modelos de simulación estocástica, ya que estos alcanzan una incertidumbre de alto nivel, esto debido a que los sistemas que se analizan para el abastecimiento de productos agrícolas son considerados sistemas complejos, en donde existe alto grado de variabilidad del comportamiento de las variables que definen su funcionamiento. Esto no quiere decir que los estudios aborden en su totalidad variables estocásticas, debido a que diversidad de autores con el propósito de facilitar el análisis y obtención de resultados buscan trabajar con modelos determinísticos, por lo tanto, establecen estrategias para controlar variables consideradas estocásticas como si fuesen determinísticas.

El $70 \%$ de los autores emplean modelos heurísticos para la solución de problemáticas asociadas a las agro-cadenas, y el $30 \%$ restante se ven obligados a emplear meta-heurísticas dada complejidad de los problemas que abordan. El enfoque de solución más allá de ser una elección de los autores se puede convertir en una obligación dependiendo de la complejidad del modelo planteado, conviniendo generalmente las de tipo heurística, ya que, los resultados suelen ser más exactos en un tiempo de cálculo más corto que las meta-heurísticas.

Analizando el tipo de programación que se emplea para el estudio de cadenas agrícolas se tiene que, de los 200 artículos revisados, el 50\% corresponde a programación lineal entera mixta y el $30,5 \%$ a programación lineal siendo las más representativas. Este tipo de programaciones son útiles para la solución de problemas de programación de producción, de distribución de productos, de ruteo de vehículos, de localización de instalaciones, de distribución de plantas, gestión de proveedores, entre otros facilitando la toma decisiones acertadas y oportunas.

De acuerdo con el número de funciones objetivo usadas en los modelos matemáticos, se determina que el $75 \%$ de las investigaciones se componen de un solo objetivo, ya que, se reconoce que al proponer más funciones objetivo aumenta el número de variables y a su vez el grado de dificultad para conseguir una solución; sin embargo, en la literatura se evidencia la necesidad de la creación de modelos matemáticos más complejos que busquen el control de mayor número de variables de forma "simultanea", esto para poder entender el comportamiento de las agro-cadenas y a su vez facilitar la toma de decisiones. Se tiene que las variables más estudiadas fueron los costos, lo que ha permitido a diversidad de autores la medición del desempeño de las agro-cadenas; sin embargo, existe evidencia de la medición de la cadena a partir de otras variables como el tiempo de entrega (79 investigaciones), la evaluación del impacto ambiental con la reducción de gases efecto invernadero (50 investigaciones), la calidad (51 investigaciones) y la temperatura (17 investigaciones) que afectan la logística de entrega a los diferentes clientes, y los niveles de inventario (56 trabajos) permitiendo el control productos perecederos (40 investigaciones).

Durante el análisis por subsector se encontró que, el mayor porcentaje de las investigaciones se centran en el sector de la agricultura con un $80 \%$ (159 investigaciones) debido que en este sector se concentran la mayor parte de productos frescos de consumo habitual como frutas, verduras, cereales, legumbres, hortalizas y cítricos, además de incluir el procesamiento de residuos agrícolas para la producción de bio-combustibles al sector energético, esto era de esperarse debido a que la investigación se centró en el sector agrícola; sin embargo, existen otras investigaciones consideradas relevantes que se desarrollan en otros sectores como la ganadería con el $8 \%$, silvicultura y otros con el $5 \%$. Existe potencial en materia de generación de nuevo conocimiento en estos sub sectores, dado que no han sido muy estudiados por la comunidad científica.

De acuerdo con los resultados, el uso de herramientas tecnológicas tiene una gran importancia para el desarrollo de la modelación matemática, son indispensables para la consecución de información de datos reales de forma rápida y precisa. La mayor parte de las investigaciones hicieron uso del GPS (Global Positioning System) con el $26,1 \%$, ya que esta herramienta permite la ubicación geográfica de almacenes, plantas, centros de acopio, clientes, entre otras locaciones. Es importante tener en cuenta que este tipo de herramientas puede facilitar la toma de datos en investigaciones futuras asociadas a las agro-cadenas.

Finalmente, 197 investigaciones desarrollan trabajo computacional debido a la complejidad de los modelos que se desarrollan. Se requiere la implementación de softwares de optimización para 
dar una solución factible y rápida a los modelos, permitiendo el control y medición de diversidad de variables desde diferentes enfoques de programación, por tanto, este tipo de herramientas siempre deben estar presentes en el desarrollo de modelos matemáticos. Existen programas computacionales de mayor uso por los

\section{REFERENCIAS BIBLIOGRAFICAS}

[1] FAO, 2017. [En línea]. Available: http://www.fao.org/3/a-17658s.pdf.

[2] B. Manos, P. Chatzinikolaou y F. Kiomourtzi, "Sustainable Optimization of Agricultural Production,» APCBEE Procedia, 2013.

[3] D. Garcia Sales, R. Porto Solano, . R. Simancas, M. Molina Correa y . H. Hernández Palma, "Sistemas de pronósticos agrícolas, una,» Producción mas limpia, pp. 142-149, 2016.

[4] A. Rong, R. Akkerman y M. MartinGrunow, «An optimization approach for managing fresh food quality throughout,» International Journal of Production Economics, pp. 421-429, 2009.

[5] H. Allaoui, Y. Guo, A. Choudhary y J. Bloemhof, "Sustainable agro-food supply chain design using two-stage hybrid multiobjective decision-making approach," Computers and Operations Research, 2016.

[6] M. Lütke Entrup, «Advanced Planning in Fresh Food Industries,» METRO, pp. XIV, 240, 2005.

[7] OCDE/FAO, «Guía OCDE-FAO para las cadenas de suministro responsable en el sector agrícola,» Éditions OCDE, Paris, 2017.

[8] R. García Flores, A. Higgins, D. Prestwidge y S. McFallan, "Optimal location of spelling yards for the northern Australian beef supply chain," Computers and Electronics in Agriculture, pp. 134-145, 2014.

[9] Z. Sazvar, M. Rahmani y K. Govindan, «A sustainable supply chain for organic, conventional agro-food products: The role of demand substitution, climate change and public health,» Journal of Cleaner Production, 2018.

[10] S. Bairamzadeh, M. MohammadSaidiMehrabad y M. S. Pishvaee, "Modelling different types of uncertainty in biofuel supply network design and planning: $A$ robust investigadores, ya sea por la cantidad y calidad de herramientas que los componen, entendimiento de la interfaz con el usuario o velocidad del proceso, conformando el selecto grupo de CPLEX, MATLAB, GAMS y LINGO que sin duda hacen que el software sea una parte vital en la solución de modelos.

optimization approach,» Renewable Energy, pp. 500-517, 2018.

[11] M. Soysal, J. M. Bloemhof Ruwaard y J. vanderVorst, «Modelling food logistics networks with emission considerations The case of an international beef supply chain,» International Journal of Production Economics, pp. 57-70, 2013.

[12] V. Kryvoruchko, A. Machmüller, V. VitomirBodiroza, B. Amon y T. Amon, "Anaerobic digestion of by-products of sugar beet and starch potato processing, » Biomass and Bioenergy, pp. 620-627, 2009.

[13] F. Lanuzza, F. Mondello y F. Tripodo, «Routes towards Enviromental Sustainability,» 2014.

[14] F. De Menna, R. A. Malagnino, M. Vittuari, A. Segrè, G. Molari, P. Deligios, S. Solinas y L. Ledda, «Optimization of agricultural biogas supply chains using artichoke byproducts in existing plants,» Agricultural Systems, pp. 137-146, 2018.

[15] R. Junqueira y R. Morabito, «Production and logistics planning considering circulation taxes in a multi-plant seed corn company,» 2012.

[16] D. Foo, R. Tan, H. Loong Lam, M. . K. Abdul Aziz y J. Jaromír Klemes, «Robust models for the synthesis of flexible palm oil-based regional bioenergy supply chain,» Energy, pp. 68-73, 2013.

[17] T. López, J. Herrera, F. González, G. Cid y Y. Chaterlán, «Eficiencia de un modelo de simulación de cultivo,» Ciencias Tecnicas Agropecuarias, pp. 1-6, 2009.

[18] X. Liu, Y. Bai y J. Chen, "An intermodal transportation geospatial network modeling for containerized soybean shipping,» Journal of Ocean Engineering and Science, 2017.

[19] M. M. Morales Chávez, J. Soto Mejía y W. Sarache, «A mixed-integer linear programming model for harvesting, loading,» 2016. 
[20] C. D. Rocco y R. Morabito, «Production and logistics planning in the tomato processing industry: A conceptual scheme and mathematical model,» Computers and Electronics in Agriculture, 2016.

[21] M. M. Morales Chávez, W. Sarache y Y. Costa, "Towards a comprehensive model of a biofuel supply chain,» Elsevier Ltd., 2018.

[22] H. Etemadnia, S. Goetz, P. Canning y M. Sadegh Tavallali, «Optimal wholesale facilities location within the fruit and vegetables supply chain with bimodal transportation options: An LP-MIP heuristic approach,» European Journal of Operational Research, 2015.

[23] M. Akbari Kaasgari, D. Mohammad Imani y M. Mahmoodjanloo, «Optimizing a Vendor Managed Inventory (VMI) Supply Chain for Perishable Products by Considering Discount: Two Calibrated Meta-heuristic Algorithms,» Computers and Industrial Engineering, 2016.

[24] K. Lamsal, P. Jones y B. Thomas, "Optimizing a location allocation-inventory problem in a two-echelon supply chain network: A modified Fruit Fly optimization algorithm,» Computers and Industrial Engineering, 2015.

[25] S. M. Mousavi, N. Alikar, S. T. Akhavan y A. Bahreininejad, «Optimizing a location allocation-inventory problem in a two-echelon supply chain network: A modified Fruit Fly optimization algorithm," Computers and Industrial Engineering, 2015.

[26] E. Alfonso, J. Montoya y E. Gutiérrez, "Modeling reverse logistics process in the agro-industrial sector: The case of the palm oil supply chain,» Applied Mathematical Modelling, 2013.

[27] J. Springael, . A. Paternoster y J. Braet, «Reducing postharvest losses of apples: Optimal transport routing (while minimizing total costs),» Computers and Electronics in Agriculture, 2018.

[28] D. Mogale, S. Krishna y M. Kumar, «An MINLP model to support the movement and storage decisions of the Indian food grain supply chain,» Control Engineering Practice, 2018.

[29] R. Tordecilla Madera, «A robust design for a Colombian dairy cooperative's milk storage and refrigeration logistics system using binary programming,» Int. J.Production Economics, 2017.

[30] S. Validi, A. Bhattacharya y P. Byrne, «A case analysis of asustainable food supply chain distribution system-A multi-objective approach,» International Journal of Production Economics, pp. 71-87, 2014.

[31] M. Soysal, J. Bloemhof, R. Haijema y J. Vander Vorst, «Modeling an Inventory Routing Problem for perishable products with environmental considerations and demand uncertainty," International Journal of Production Economics, pp. 118-133, 2015.

[32] A. Dooley, W. Parker y H. Blair, «Modelling of transport costs and logistics for on-farm milk segregation in New Zealand dairying,» Computers and Electronics in Agriculture, pp. 75-91, 2005.

[33] T. Paksoy, N. Pehlivan y E. Özceylan, «Application of fuzzy optimization to a supply chain network design: A case study of an edible vegetable oils manufacturer, » Applied Mathematical Modelling, 2011.

[34] H. Gunnarsson, M. Roonnqvist y D. Carlsson, «Economic aspects of the cotton-stalk biomass logistics and comparison of supply chain methods," Biomass and Bioenergy, 2006.

[35] R. Akkerman, P. Farahani y M. Grunow, "Quality, safety and sustainability in food distribution,» OR Spectrum, pp. 863-904, 2010.

[36] A. Hiassata, A. Diabat y I. Rahwan, «A genetic algorithm approach for location-inventoryrouting problemwith perishable products," Journal of Manufacturing Systems, pp. 93103, 2016.

[37] J. Zhang, «The water-food-energy nexus optimization approach to combat,» Elservier, 2017.

[38] L. Coelho y G. Laporte, «Optimal joint replenishment, delivery and inventory management policies forperishable products," Computers and Operations Research, pp. 42-52, 2014.

[39] G. Behzadi, M. J. O'Sullivan, T. L. Olsen, F. Scrimgeour y A. Zhang, "Robust and resilient strategies for managing supply disruptions in an agribusiness, " International Journal of Production Economics, 2017. 
[40] B. Bilgen y I. Ozkarahan, «A mixed-integer linear programming model for bulk grain blending and shipping,» International Journal of Production Economics, 2007.

[41] S. Mirzaei y A. Seifi, "Considering lost sale in inventory routing problems for perishable goods,» Computers and Industrial Engineering, pp. 213-227, 2015.

[42] G. Claassen y T. Hendriks, "An application of Special Ordered Sets to a periodic,» European Journal of Operational Research, 2006.

[43] J. Arnaout y M. Maatoukw, "Optimization of quality and operational costs through improved scheduling of harvest operations,» International Transactions in operational research, 2009.

[44] L. Maiyar, J. Thakkar, A. Awasthi y M. Kumar, «Development of an Effective Cost Minimization Model for Food Grain Shipments,» IFAC, 2015.

[45] D. Shen, K. Lai, S. Leung y L. Liang, "Modelling and analysis of inventory replenishment for perishable agricultural products with buyer-seller collaboration,» International Journal of Systems Science, pp. 1207-1217, 2011.

[46] C. Ing Hsu y W. Ting Chen, "Optimizing fleet size and delivery scheduling for multitemperature food distribution,» Applied Mathematical Modelling, pp. 1077-1091, 2013.

[47] C. Sel, B. Bilgen, J. Bloemhof y J. van der Vorst, «Multi-bucket optimization for integrated planning and scheduling inthe perishable dairy supply chain,» Computers and Chemical Engineering, pp. 59-73, 2015.

[48] M. Cortés y M. León, "Generalidades sobre Metodología,» Universidad Autonoma del Carmen, pp. 1-105, 2004.

[49] O. Ahumada y R. Villalobos, «Application of planning models in the agri-food supply chain A review, European Journal of Operational Research, pp. 1-20, 2009.

[50] B. Gebreslassie, Y. Yao y F. You, "Design Under Uncertainty of Hydrocarbon Biorefinery Supply Chains: Multiobjective Stochastic Programming Models, Decomposition Algorithm, and a Comparison Between CVaR and Downside Risk," American Institute of Chemical Engineers, pp. 2155-2179, 2012.
[51] H. Seyyedhasani y J. Dvorak, «Using the Vehicle Routing Problem to reduce field completion times with multiple machines,» Computers and Electronics in Agriculture, pp. 142-150, 2016.

[52] S. Araújo Reis y J. E. Leal, «A deterministic mathematical model to support temporal and spatial decisions of the soybean supply chain,» Journal of Transport Geography, pp. 48-58, 2015.

[53] L. M. Dos Santos, A. Costa, M. Arenales y R. Santos, "Sustainable vegetable crop supply problem,» European Journal of Operational Research, 2009.

[54] C. Nguyen, M. Dessouky y A. Toriello, "Consolidation strategies for the delivery of perishable products," Transportation Research, pp. 108-121, 2014.

[55] D. Mogale, S. Krishna y M. Kuma, «Two Stage Indian Food Grain Supply Chain Network Transportation-Allocation Model,» IFAC, 2016.

[56] K. Kittilertpaisan y S. Pathumnakul, «Integrating a multiple crop year routing design for sugarcane harvesters to plant a new crop," Computers and Electronics in Agriculture, 2017.

[57] I. Tatsiopoulos y A. Tolis, «Economic aspects of the cotton-stalk biomass logistics and comparison of supply chain methods," Biomass and Bioenergy, 2002.

[58] A. Cheraghalipoura, M. Mahdi y M. Hajiaghae, «A bi-objective optimization for citrus closedloop supply chain using Pareto-based algorithms,» Applied Soft Computing, 2018.

[59] Z. Guan y A. Philpott, «A multistagestochasticprogrammingmodelforth eNewZealand,» Elservier, 2009.

[60] B. Gardasa, R. Rautb y B. Narkhedec, «Modeling causal factors of post-harvesting losses in vegetable and fruit,» 2017.

[61] D. Ambrosino y A. Sciomachen, «A food distribution network problem: a case study,» Journal of Management Mathematics, 2004.

[62] N. Azi, M. Gendreau y J. Yvespotvin, «An exact algorithm for a single-vehicle routing problem with time windows and multiple routes., European Journal of Operational Research, 2006. 
[63] H. Gunnarson, M. Roonnqvist y D. Carlsson, «A combined terminal location and ship routing problem, » Journal of the Operational Research Society, 2006.

[64] K. Piewthongngam, P. Vijitnopparat, S. Pathumnakul, S. Chumpatong y $\mathrm{M}$. Duangjinda, "System dynamics modelling of an integrated pig production supply chain,» Biosystems Engineering, 2014.

[65] K. Piewthongngam, S. Pathumnakul y K. Setthanan, «Application of crop growth simulation and mathematical modeling to supply chain management in the Thai sugar industry,» Agricultural Systems, pp. 58-66, 2009.

[66] D. Agustina, C. Lee y R. Piplani, «Vehicle scheduling and routing at a cross docking center for food supply chains,» International Journals of Production Economics, pp. 29-41, 2014.

[67] P. Amorim, B. Almada, B. Póvoa y I. Grossmann, "Combining Supplier Selection and Production-Distribution Planning in Food Supply Chain," European Symposium on Computer Aided Process Engineering, 2014.

[68] S. Atallah, M. Gómez y T. Björkman, "Localization effects for a fresh vegetable product supply chain: Broccoli in the eastern United States,» Food Policy, pp. 151-159, 2014.

[69] K. Govindan, A. Jafarian, R. Khodaverdi y K. Devika, «Two-echelon multiple-vehicle location-routing problem with time windows for optimization of sustainable supply chain network of perishable food,» International Journal Of Production Economics, pp. 9-28, 2013.

[70] G. Lopez, J. L. Martinez, J. Cavazos y Y. Mayett, "Mezcal Supply Chain in Zacatecas State. Current situation and perspectives,» Contaduria y administración, pp. 227-252, 2012.

[71] Y. Kazancoglu, Y. D. Ozkan y M. Ozbiltekin, «Minimizing losses in milk supply chain with sustainability: An example from an emerging economy,» Resources, Conservation and Recycling, 2018.

[72] F. Dabbene, P. Gay y N. Sacco, "Optimisation of fresh-food supply chains in uncertain environments, Part I: Background and methodology,» Biosystems Engineering, 2007.

[73] Z. Sazvar, M. Sepehri y A. Baboli, «A Multiobjective Multi-Supplier Sustainable Supply Chain with Deteriorating Products, Case of Cut Flowers," International Federation of Automatic Control, pp. 1638-1643, 2016.

[74] J. Carvajal, W. Sarache y Y. Costa, "Addressing a robust decision in the sugarcane supply chain: Introduction of,» Elsevier, 2018.

[75] A. Mohammed y Q. Wang, «The fuzzy multiobjective distribution planner for a green meat supply chain,» International Journal of Production Economics, pp. 47-58, 2016.

[76] A. Osvald y L. Zadnik, «A vehicle routing algorithm for the distribution of fresh vegetables and similar perishable food,» Journal of Food Engineering, 2007.

[77] S. Allen y E. Schuster, "Controlling the Risk for an Agricultural Harvest," Institute for Operations Research and the Management Sciences, 2004.

[78] J. V. Caixeta Filho, "Orange harvesting scheduling management: a case study," Journal of the Operational Research Society, 2006.

[79] J. V. Caixeta-Filho, J. M. Swaay-Neto y A. d. $P$. Wagemaker, "Optimization of the Production Planning and Trade of Lily, » 2002.

[80] R. Apaiah y E. Hendrix, «Design of a supply chain network for pea-based novel protein foods, " Journal of Food Engineering, pp. 383391, 2004.

[81] A. Biswas y B. Baran Pal, "Application of fuzzy goal programming technique to land use planning in agricultural system,» The international journal of management science, pp. 391-398, 2004.

[82] H. Chávez, K. Castillo, L. Herrera y A. Bustos, «Simulation-based multi-objective model for supply chains with disruptions in transportation,» Robotics and ComputerIntegrated Manufacturing, 2015.

[83] G. Fernández, A. Aguilar, M. Miranda, C. Moras , B. Ixmatlahua y C. Azzaro, «An expert system for predicting orchard yield and fruit quality and its impact on the Persian lime supply chain,» Engineering Applications of Artificial Intelligence, pp. 21-30, 2014. 
[84] M. Hu, Y. Chen y L. C. Huang, «A sustainable vegetable supply chain using plant factories in Taiwanese markets: A Nash-Cournot model,»Int. J.Production Economics, 2014.

[85] J. K. Gigler, E. M. Hendrix, R. A. Heesen, V. G. van den Hazelkamp y G. Meerdink, «On optimisation of agri chains by dynamic programming,» European Journal of Operational Research, 2001.

[86] S. Brusca, F. Famoso, R. Lanzafame, M. Messina y P. Monforte, «Placement optimization of biodiesel production plant by means of centroid mathematical method," Energy Procedia, pp. 353-360, 2017.

[87] J. C. Ferrer, A. Mac , S. Maturana, S. Toloza y J. Vera, "An optimization approach for scheduling wine grape harvest operations,» Int. J.Production Economics, 2007.

[88] H. Stellingwerf, A. Kanellopoulo, J. van der Vorst y J. Bloemhof, «Reducing $\mathrm{CO} 2$ emissions in temperature-controlled roadtransportation using the LDVRP model,» Transportation Research, 2017.

[89] J. V. Caixeta Filho, J. M. Van Swaay Neto y R. L. Lopez, «Linear programming applied to the flower sector: a Gladiolus bulb production case study,» International transactions in operational research, 2000.

[90] R. Garcia, M. Martinez y F. Palacios, «Tactical optimization of the oil palm agribusiness supply chain,» Applied Mathematical Modelling, 2015.

[91] S. Pathumnakul, K. Piewthongngamb y S. Khamjanc, «Integrating a shrimp-growth function, farming skills information, and a supply allocation algorithm to manage the shrimp supply chain,» Computers and Electronics in Agriculture, 2008.

[92] D. Mogale, M. Kumar, S. Kumar y M. Kumar Tiwari, «Grain silo location-allocation problem with dwell time for optimization of food grain supply chain network,» Transportation Research, 2018.

[93] K. Moghaddam y G. DePuy, «Farm management optimization using chance constrained programming method," Computers and Electronics in Agriculture, 2011.

[94] C. Filippi, R. Mansini y E. Stevanato, «Mixed Integer Linear Programming models for optimal crop selection,» Computers and Operations Research, 2016.

[95] L. Maiyar y J. Thakkar, «A combined tactical and operational deterministic food grain transportation model: Particle swarm based optimization approach,» Computers and Industrial Engineering, 2017.

[96] A. Ridier, K. Chaib y C. Roussy, «A Dynamic Stochastic Programming model of croprotation choice to testthead option of long rotation under price and production risks,» European Journal of Operational Research, 2015.

[97] V. Venus, D. Asare, L. Tijskens, M. Weir, C. de Bie, S. Ouedraogo, W. Nieuwenhuis, S. Wesselman, G. Cappelli y E. Smaling, «Development and validation of a model to estimate postharvest losses during transport of tomatoes in West Africa,» Computers and Electronics in Agriculture, 2012.

[98] W. E. Soto-Silva, E. Nadal Roig, M. C. González Araya y L. M. Pla Aragones, "Operational research models applied to the fresh fruit supply chain,» EuropeanJournalofOperationalResearch, pp. 345-355, 2016.

[99] R. Masson, N. Lahrichi y L. Martin, «A twostage solution method for the annual dairy transportation problem, " European Journal of Operational Research, 2015.

[100] J. Qian, B. Fan, X. Wu, S. Han, S. Liu y X. Yang, "Comprehensive and quantifiable granularity: A novel model to measure agrofood traceability,» Food Control, 2016.

[101] I. Awudu y J. Zhang, «Stochastic production planning for a biofuel supply chain under demand and price uncertainties,» Applied Energy, 2012.

[102] A. Baghalian, S. Rezapour y R. Zanjirani, «Robust supply chain network design with service level against disruptions and demand uncertainties: A real-life case,» European Journal of Operational Research, 2012.

[103] O. Ahumada, R. Villalobos y N. Mason, «Tactical planning of the production and distribution of fresh agricultural products under uncertainty,» Agricultural Systems, pp. 17-26, 2012.

[104] O. Ahumada y R. Villalobos, «A tactical model for planning the production and distribution of 
fresh produce,» Annals of Operations Research, 2009.

[105] B. Tan y N. Çömden, «Agricultural planning of annual plants under demand, maturation, harvest, and yield risk, " European Journal of Operational Research, 2012.

[106] H. Chen, M. S. Chang y C. F. Hsueh, "Production scheduling and vehicle routing with time windows for perishable,» Computers and Operations Research, 2008.

[107] J. Kim, M. Realff y J. Lee, «Optimal design and global sensitivity analysis of biomass supply chain networks for biofuels under uncertainty,» Computers and Chemical Engineering, 2011.

[108] S. Mirzapour, H. Malekly y M. Aryanezhad, «A multi-objective robust optimization model for multi-product multi-site aggregate production planning in a supply chain under uncertainty,» Int. J.Production Economics, 2011.

[109] C. Bohle, S. Maturana y J. Vera, «A robust optimization approach to wine grape harvesting scheduling,» European Journal of Operational Research, 2008.

[110] W. Jang y C. Klein, «Supply chain models for small agricultural enterprises,» Annals of Operations Research, 2009.

[111] K. Widodo, H. Nagasawa, K. Morizawa y M. Ota, «A periodical flowering-harvesting model for delivering agricultural fresh products,» European Journal of Operational Research, 2006.

[112] W. feng, Z. Guan y V. Whitaker, «Optimizing yield distribution under biological and economic constraints: Florida strawberries as a model for perishable commodities," Agricultural Systems, 2015.

[113] K. Bakhrankova, K. Midthun y K. Uggen, «Stochastic optimization of operational production planning for fisheries,» Fisheries Research, pp. 147-153, 2014.

[114] E. Colin, «Mathematical programming accelerates implementation of agro-industrial sugarcane complex, European Journal of Operational Research, 2008.

[115] W. Chen, J. Li y X. Jin, «The replenishment policy of agri-products with stochastic demand in integrated agricultural supply chains,» Expert SystemsWith Applications, 2015.
[116] H. D. O. Florentino, A. D. De Lima, L. R. De Carvalho, A. R. Balbo y T. P. Donadon Homem, "Multiobjective 0-1 integer programming for the use of, " INTERNATIONAL TRANSACTIONS IN OPERATIONAL RESEARCH, 2011.

[117] A. Duarte, W. Sarache y Y. Acosta, «A facilitylocation model for biofuel plants: Applications in the Colombian context,» Energy, 2004.

[118] M. Grunow, H. Gunthetr y R. Westinner, «Supply optimization for the production of raw sugar,» Int. J.Production Economics, 2007.

[119] A. Higgins, «Scheduling of road vehicles in sugarcane transport: A case study at an Australian sugar mill,» European Journal of Operational Research, pp. 987-1000, 2004.

[120] S. Jena y M. Poggi, "Harvest planning in the Brazilian sugar cane industry via mixed integer programming,» European Journal of Operational Research, 2013.

[121] Z. Jiao, A. Higgins y D. Prestwidge, «An integrated statistical and optimisation approach to increasing sugar production within a mill region,» Computers and Electronics in Agriculture, 2005.

[122] W. Chen, J. Li y X. Jin, «An improved evolutionary algorithm for solving multiobjective crop planning models,» Expert SystemsWith Applications, 2015.

[123] S. Wiedenmann y J. Geldermann, «Supply planning for process ors of agricultural raw materials, » European JournalofOperationalResearch, pp. 606-619, 2014.

[124] J. A. Orjuela Castro, L. A. Sanabria Coronado y A. M. Peralta Lozano, "Coupling facility location models in the supply chain of perishable fruits, Research in Transportation Business \& Management, 2017.

[125] S. Tabrizi, S. Hassan y A. Ahmadi, «Modelling three-echelon warm-water fish supply chain: A bi-level optimization approach under NashCournot equilibrium,» Applied Soft Computing, 2017.

[126] N. Asgari, R. Zanjirani, H. Rashidi y M. Sajadieh, «Developing model-based software to optimise wheat storage and transportation: A real-world application,» Applied Soft Computing, 2012. 
[127] D. Mogale, S. Krishna, F. Garcia y M. Kumar, «Bulk wheat transportation and storage problem of Public Distribution System," Computers and Industrial Engineering, 2016.

[128] M. Gholamian y A. Hakim, «Integrated network design of wheat supply chain: A real case of Iran,» Computers and Electronics in Agriculture, 2017.

[129] P. Amorin, H. Gunther y B. Almada, «Multiobjective integrated production and distribution planning of perishable products, » International Journal Of Production Economics, pp. 89-101, 2012.

[130] J. Annetts y E. Audsley, «Multiple objective linear programming for environmental farm planning,» Journal of the Operational Research Society, 2002.

[131] L. Catalá, S. Moreno, A. Blanco y A. Bandoni, «A bi-objective optimization model for tactical planning in the pome fruit industry supply chain,» Computers and Electronics in Agriculture, pp. 128-141, 2016.

[132] L. Catalá, G. Duran, A. Blanco y A. Bandoni, «Mathematical model for strategic planning optimization in the pome fruit industry,» Agricultural Systems, pp. 63-71, 2012.

[133] Y. Ampatzidis, S. Vougioukas, M. Whiting y Q. Zhang, «Applying the machine repair model to improve efficiency of harvesting fruit,» Biosystems Engineering, 2013.

[134] M. Bortolini, M. Faccio, E. Ferrari, M. Gamberi y F. Pilati, «Fresh food sustainable distribution: cost, delivery time and carbon footprint three-objective optimization, 》 Journal of Food Engineering, pp. 1-12, 2016.

[135] A. Banasik, A. Kanellopoulus, G. Claassen, J. Bloemhof y G. Vandervorsh, "Closing loops in agricultural supply chains using multiobjective optimization: A case study of an industrial mushroom supply chain,» Int. J.Production Economics, 2016.

[136] H. Grillo, M. Alemany, A. Ortiz y V. Fuerte, "Mathematical modelling of the orderpromising process for fruit supply chains considering the perishability and subtypes of products,» Applied Mathematical Modelling, 2017.

[137] M. Miranda, C. Azzaro y A. Aguilar, «A green supply chain network design framework for the processed food industry: application to the orange juice agrofood cluster,» Computers and Industrial Engineering, 2017.

[138] O. Boyabatli, P. Kleindorfer y S. Koontz, «Procurement Risk Management in Beef Supply Chains,» Industrial Aplications, 2012.

[139] A. Blanco, G. Masini, N. Petracci y A. Bandoni, «Model Predictive Control Based Planning in the Fruit Industry,» European Symposium on Computer Aided Process Engineering, 2008.

[140] R. Sarker y T. Ray, «An improved evolutionary algorithm for solving multiobjective crop planning models," Computers and Electronics in Agriculture, 2009.

[141] X. Zeng, S. Kang, F. Li, L. Zhang y P. Guo, «Fuzzy multi-objective linear programming applying to crop area planning,» Agricultural Water Management, 2010.

[142] S. Osama, M. Elkholy y R. Kansoh, "Optimization of the cropping pattern in Egypt,» Alexandria Engineering Journal, 2017.

[143] E. Lopez, J. Orengo, J. Tarjuelo, A. Martinez y A. Dominguez, «Development of a directsolution algorithm for determining theoptimal crop planning of farms using deficit irrigation, » Agricultural Water Management, 2016.

[144] N. Garcia, A. Lozano y Y. Rios, «A crop planning and real-time irrigation method based on site-specific management zones and linear programming," Computers and Electronics in Agriculture, pp. 20-28, 2014.

[145] J. Adeyemo y F. Otieno, «Differential evolution algorithm for solving multi-objective crop planning model,» Agricultural Water Management, 2010.

[146] S. Saranwong y C. Likasiri, «Bi-level programming model for solving distribution center problem: A case study in Northern Thailand's sugarcane management,» Computers and Industrial Engineering, 2016.

[147] H. Flores, R. Villalobos, O. Ahumada, M. Uchanski, C. Meneses y O. Sanchez, «Use of supply chain planning tools for efficiently placing small farmers into high-value, vegetable markets,» Computers and Electronics in Agriculture, 2019.

[148] R. Tieppo, T. Liborio, M. Milan, C. Gron y D. Bochtis, «Modeling cost and energy demand in agricultural machinery fleets for soybean 
and maize cultivated using a no-tillage system,» Computers and Electronics in Agriculture, 2019.

[149] J. Monge y S. Wakelin, "Geographicallyexplicit, dynamic partial equilibrium model of regional primary value chains - Mathematical formulation and application to forestry in the Northland region of New Zealand," Computers and Electronics in Agriculture, 2019.

[150] N. Brulard, V. D. Cung y N. Catusse, «Client selection and combination for farm perishable products," Computers and Electronics in Agriculture, 2017.

[151] H. Seyyedhasani, J. Dvorak y E. Roemmele, «Routing algorithm selection for field coverage planning based on field shape and fleet size," Computers and Electronics in Agriculture, 2019.

[152] H. Seyyedhasani y J. Dvorak, «Dynamic rerouting of a fleet of vehicles in agricultural operations through a Dynamic Multiple Depot Vehicle Routing Problem representation,» Biosystems Engineering, 2018.

[153] J. Jonkman, A. Kanellopoulos y J. Bloemhof, «Designing an eco-efficient biomass-based supply chain using a multi-actor optimisation model, 》 Journal of Cleaner Production, 2018.

[154] J. Jonkman, A. Barbosa y J. Bloemhof, "Integrating harvesting decisions in the design of agro-food supply chains, " European Journal of Operational Research, 2018.

[155] C. Herrera, F. Perez, E. Alvarez y A. Candia, "Optimization of the harvest planning in the olive oil production: A case study in Chile,» Computers and Electronics in Agriculture, 2017.

[156] X. Ma, S. Wang, S. Islam y X. Liu, "Coordinating a three-echelon fresh agricultural products supply chain considering freshness-keeping effort with asymmetric information,» Applied Mathematical Modelling, 2018.

[157] B. Sarker, B. Wu y K. Paudel, «Modeling and optimization of a supply chain of renewable biomass and biogas: Processing plant location,» Applied Energy, 2019.

[158] M. Rabbani, N. Akbarian, H. Farrokhi, S. Frederick, W. Lim y Z. Tahaei, «Developing a sustainable supply chain optimization model for switchgrass-based bioenergy production: a case study, » Journal of Cleaner Production, 2019.

[159] E. Lopez, S. Miquel y L. Pla, «Sugar cane transportation in Cuba, a case study, " European Journal of Operational Research, pp. 374-386, 2005.

[160] J. A. Orjuela, J. A. Aranda y C. E. Moreno, «Identifying trade-offs between sustainability dimensions in the supply chain of biodiesel in Colombia,» Computers and Electronics in Agriculture, 2018.

[161] M. Sharifzadeh, M. Cortada y N. Shah, "Supply chain network design and operation: Systematic decisionmaking for centralized, distributed, and mobile biofuel production using mixed integer linear programming (MILP) under uncertainty,» Biomass and Bioenergy, 2015.

[162] F. Sgarbossa y I. Russo, «A proactive model in sustainable food supply chain,» Int. J.Production Economics, 2016.

[163] S. Mejjaouli y R. Babiceanu, "Cold supply chain logistics: System optimization for realtime rerouting transportation solutions, 》 Computers in Industry, pp. 68-80, 2017.

[164] M. Saadati y S. J. Hosseininezhad, «Designing a hub location model in a bagasse-based bioethanol supply chain,» 2019.

[165] A. Banasik, A. kanellopoulos, J. Bloemhof y G. Claassen, «Accounting for uncertainty in eco-efficient agri-food supply chains: a case study for mushroom production planning," Journal of Cleaner Production, 2019.

[166] A. Golmohammadi y E. Hassini, «Capacity, pricing and production under supply and demand uncertainties with an application in agriculture,» European Journal of Operational Research, 2018.

[167] C. Craige, M. Buser, R. Frazier, S. Hiziroglu, R. Holcomb y R. Huhnke, "Conceptual design of a biofeedstock supply chain model for eastern redcedar," Computers and Electronics in Agriculture, 2015.

[168] P. E. Murillo, G. Guill, J. M. Ponce, A. J. Castro, M. Serna y L. Jimenez, «Multiobjective optimization of the supply chain of biofuels from residues of the tequila industry in Mexico," Journal of Cleaner Production, 2015. 
[169] R. Babazadeh, «Optimal design and planning of biodiesel supply chain considering nonedible feedstock,» Renewable and Sustainable Energy Reviews, 2006.

[170] Q. Li y G. Hu, «Supply chain design under uncertainty for advanced biofuel production based on bio-oil gasification,» Energy, 2014.

[171] F. Farahani, M. Grunow y R. Akkerman, «Design and operations planning of municipal food service systems., » International Journal of Production Economics, 2013.

[172] V. Gonela, J. Zhang y A. Osmani, «Stochastic optimization of sustainable industrial symbiosis based hybrid generation bioethanol supply chains,» Computers \& Industrial Engineering, 2015.

[173] H. Paulo, X. Azcue, A. Barbosa y S. Relvas, «optimization of residual forestry biomass for bioenergy production: The case study of Portugal,» Biomass and Bioenergy, 2015.

[174] D. Mogale, A. Dolgui, r. Kandhway, S. Krishna y $M$. Kumar, «A multi-period inventory transportation model for tactical planning of food grain supply chain,» Computers and Industrial Engineering, 2017.

[175] D. Bredstrom, J. Lundgren, M. Ronnqvist, D. Carlsson y A. Mason, «Supply chain optimization in the pulp mill industry-IP models, column generation and novel constraint branches,» European Journal of Operational Research, 2004.

[176] G. Devlin, A. Sosa y M. Acuña, «Solving the woody supply chain for Ireland's expanding biomass sector: a case study,» Biomass Supply Chains for Bioenergy and Biorefining, 2016.

[177] E. Nadal, L. Pia y A. Alonso, «Production planning of supply chains in the pig industry," Computers and Electronics in Agriculture, 2018.

[178] N. Vanzetti, D. Broz, G. Corsano y J. Montagna, «An optimization approach for multiperiod production planning in a sawmill,» Forest Policy and Economics, 2018.

[179] A. Boonmee y K. Sethanan, «A GLNPSO for multi-level capacitated lot-sizing and scheduling problem in the poultry industry,» European Journal of Operational Research, 2015.
[180] S. Rodriguez, L. Pla y V. Albornoz, «Modeling tactical planning decisions through a linear optimization model in sow farms, " Livestock Science, 2011.

[181] J. Zuanetti, F. Dias y A. Moura, «Application of a vendor managed inventory (VMI) system model in an animal nutrition industry,» Conference on Intelligent Computation in Manufacturing Engineering, 2017.

[182] B. Ivanov, "Optimal location of biodiesel refineries: The Bulgarian Scale,» Journal of Chemical Technology and Metallurgy, 2013.

[183] M. Ali, R. Deo, N. Downs y T. Maraseni, "Cotton yield prediction with Markov Chain Monte Carlo-based simulation model integrated with genetic programing algorithm: A new hybrid copuladriven approach," Agricultural and Forest Meteorology, 2018.

[184] W. Khamjan, S. Khamjan y S. Pathumnakul, «Determination of the locations and capacities of sugar cane loading stations in Thailand," Computers and Industrial Engineering, 2013.

[185] K. Sethanan y W. Neungmatcha, «Multiobjective Particle Swarm Optimization for Mechanical Harvester Route Planning of Sugarcane Field Operations,» European Journal of Operational Research, 2016.

[186] S. Thuankaewsing, S. Khamjan, K. Piewthongngam y S. Pathumnakul, «Harvest scheduling algorithm to equalize supplier benefits: A case study from the Thai sugar cane industry, " Computers and Electronics in Agriculture, 2014.

[187] O. Dowson, A. Philpott, A. Mason y A. Downward, «A multi-stage stochastic optimization model of a pastoral dairy farm,» European Journal of Operational Research, 2018.

[188] S. Mohammad, Y. Bai, G. Maia, Y. Ouyang y L. Rodriguez, "Grain supply chain network design and logistics planning for reducing post-harvest loss,» Biosystems Engineering, 2016.

[189] K. Darby, S. Barker, E. Audsley y D. Parsoms, «A two-stage stochastic programming with recourse model for determining robust planting plans in horticulture, " Journal of the Operational Research Society, 2000.

[190] S. Foog, C. Goh, C. Supramaniam y D. Ng, «Input-output optimisation model for sustainable oil palm plantation development,» 
Sustainable Production and Consumption, 2018.

[191] N. Shabani y T. Sowlati, «A mixed integer non-linear programming model for tactical value chain optimization of a wood biomass power plant,» Applied Energy, 2012.

[192] L. Sha Sha, L. Yan Sui, L. Hua y G. Xing, «Agricultural Production Structure Optimization: A Case Study of Major Grain Producing Areas, China,» Journal of Integrative Agriculture, 2012.

[193] Y. Costa, A. Duarte y W. Sarache, «A decisional simulation-optimization framework for sustainable facility location of a biodiesel plant in Colombia,» Journal of Cleaner Production, 2017.

[194] H. Khajarpanah, M. Saman Pishvaee y S. Mohammad, «A risk averse cross-efficiency data envelopment analysis model for sustainable switchgrass cultivation location optimization," Industrial Crops \& Products, 2017.

[195] K. Lamsal, P. C. Jones y B. W. Thomas, «Harvest logistics in agricultural systems with multiple, independent producers and no onfarm storage,» Computers and Industrial Engineering, 2015.

[196] O. Ahumada y R. Villalobos, «Operational model for planning the harvest and distribution of perishable a gricultural products,» Int. J.Production Economics, 2011.

[197] H. Flores y R. Villalobos, «A modeling framework for the strategic design of local fresh-food syst,» Agricultural Systems, pp. 115, 2018.

[198] F. Wouda, P. Van Beerk, J. Van der Vorst y H. Tacke, «An application of mixed-integer linear programming models on the redesign of the supply network of Nutricia Dairy \& Drinks Group in Hungary,» OR Spectrum, 2002.

[199] P. Jones, T. Lowe, R. Traub y G. Kegler, «Matching Supply and Demand: The Value of a Second Chance in Producing Hybrid Seed Corn,» Manufacturing \& Service Operations Management, 2001.

[200] P. Jones, G. Kegler, T. Lowe y R. Traub, "Managing the Seed-Corn Supply Chain at Syngenta,» Institute for Operations Research and the Management Sciences, 2003.
[201] B. Kazaz, «Production Planning Under Yield and Demand Uncertainty with YieldDependent Cost and Price,» Manufacturing \& Service Operations Management, 2004.

[202] A. Blanco, G. Masini, N. Petracci y J. Bandoni, «Operations management of a packaging plant in the fruit industry," Journal of Food Engineering, 2004.

[203] S. Hester y O. Cacho, «Modelling apple orchard systems,» Agricultural Systems, 2002.

[204] P. Amorim y B. Almada, «The impact of food perishability issues in the vehicle routing problem,» Computers and Industrial Engineering, 2013.

[205] J. P. Anaout y M. Maatoukw, «Optimization of quality and operational costs through improved scheduling of harvest operations,» International Transactions in operational research, 2009.

[206] J. Blackburn y G. Scudder, «Supply Chain Strategies for Perishable Products: The Case of Fresh Produce,» Production and Operations Management Society, 2008.

[207] E. Cittadini, M. Lubbers, N. Ridder, H. Van Keulen y G. Claassen, «Exploring options for farm-level strategic and tactical decisionmaking in fruit production systems of South Patagonia, Argentina, Agricultural Systems, 2008.

[208] V. R. Ghezavati, S. Hooshyar y R. Tavakkoli Moghaddam, «A Benders' decomposition algorithm for optimizing distribution of perishable products considering postharvest biological behavior in agri-food supply chain: a case study of tomato,» 2015.

[209] J. R. Munhoz y R. Morabito, «Optimization approaches to support decision making in the production planning of a citrus company: $A$ Brazilian case study,» Computers and Electronics in Agriculture, 2014.

[210] L. M. Tijskens, T. Unuk, R. C. Okello, A. M. Wubs, V. Sustar, D. Sumak y R. E. Schouten, «From fruitlet to harvest: Modelling and predicting size and itsdistributions for tomato, apple and pepper fruit.,» Scientia Horticulturae, 2016.

[211] M. Varsei y S. Polyakovskiy, «Sustainable supply chain network design: A case of the wine industry in Australia,» International Journal of Management Science, 2015. 
[212] B. Vitoriano, M. Ortuno, B. Recio, F. Rubio y A. Alonso, "Two alternative models for farm management: Discrete versus continuous time horizon," Elservier, 2001.

[213] D. Zhou, A. Leck, D. Bochtis y C. Sorensen, "Simulation model for the sequential in-field machinery operations in a potato production system," Computers and Electronics in Agriculture, 2015. 